\title{
The middle Holocene climatic records from Arabia: Reassessing lacustrine environments, shift of ITCZ in Arabian Sea, and impacts of the southwest Indian and African monsoons
}

\author{
Yehouda Enzel $^{\mathrm{a}, *}$, Yochanan Kushnir $^{\mathrm{b}}$, Jay Quade ${ }^{\mathrm{c}}$ \\ a The Fredy and Nadine Herrmann Institute of Earth Sciences, The Hebrew University of Jerusalem, Givat Ram, Jerusalem 91904, Israel \\ b Lamont-Doherty Earth Observatory, Columbia University, 61 Route 9W, PO Box 1000, Palisades, NY 10964-8000, USA \\ ${ }^{c}$ Department of Geosciences, University of Arizona, 1040 E. 4th Street, Tucson, AZ 85721, USA
}

\section{A R T I C L E I N F O}

\section{Article history:}

Received 14 July 2014

Received in revised form 3 March 2015

Accepted 11 March 2015

Available online 21 March 2015

\section{Keywords:}

Arabia

Indian monsoon

African monsoon

Holocene paleoclimate

lake

ITCZ

Somali jet

\begin{abstract}
A B S T R A C T
A dramatic increase in regional summer rainfall amount has been proposed for the Arabian Peninsula during the middle Holocene (ca. 9-5 ka BP) based on lacustrine sediments, inferred lake levels, speleothems, and pollen. This rainfall increase is considered primarily the result of an intensified Indian summer monsoon as part of the insolation-driven, northward shift of the boreal summer position of the Inter-Tropical Convergence Zone (ITCZ) to over the deserts of North Africa, Arabia, and northwest India.

We examine the basis for the proposed drastic climate change in Arabia and the shifts in the summer monsoon rains, by reviewing paleohydrologic lacustrine records from Arabia. We evaluate and reinterpret individual lakebasin status regarding their lacustrine-like deposits, physiography, shorelines, fauna and flora, and conclude that these basins were not occupied by lakes, but by shallow marsh environments.

Rainfall increase required to support such restricted wetlands is much smaller than needed to form and maintain highly evaporating lakes and we suggest that rainfall changes occurred primarily at the elevated edges of southwestern, southern, and southeastern Arabian Peninsula. These relatively small changes in rainfall amounts and local are also supported by pollen and speleothems from the region. The changes do not require a northward shift of the Northern Hemisphere summer ITCZ and intensification of the Indian monsoon rainfall. We propose that (a) latitudinal and slight inland expansion of the North African summer monsoon rains across the Red Sea, and (b) uplifted moist air of this monsoon to southwestern Arabia highlands, rather than rains associated with intensification of Indian summer monsoon, as proposed before, increased rains in that region; these African monsoon rains produced the modest paleo-wetlands in downstream hyperarid basins. Furthermore, we postulate that as in present-day, the ITCZ in the Indian Ocean remained at or near the equator all year round, and the Indian summer monsoon, through dynamically induced air subsidence, can reduce rather than enhance summer rainfall in the Levant and neighboring deserts, including Arabia. Our summary suggests a widening to the north of the latitudinal range of the rainfall associated with the North African summer monsoon moisture crossing the Red Sea to the east. We discuss other mechanisms that could have potentially contributed to the formation and maintaining of the modest paleo-wetlands.
\end{abstract}

(C) 2015 Elsevier B.V. All rights reserved.

\section{Contents}

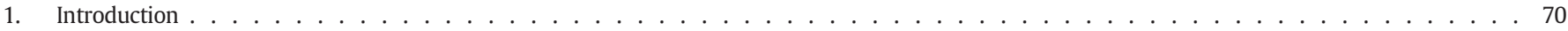

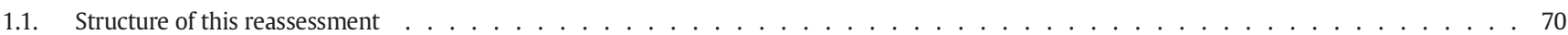

2. Geographical and Meteorological constraints on Arabian rainfall ． . . . . . . . . . . . . . . . . . . . . . . 70

2.1. Annual and seasonal rainfall distribution . . . . . . . . . . . . . . . . . . . . . . . . . . . 70

2.2. Synoptic climatology of Arabia rainfall . . . . . . . . . . . . . . . . . . . . . . . . . . . . . 70

2.2.1. Summer rainfall in Arabia . . . . . . . . . . . . . . . . . . . . . . . . . . . . . . . . . . . . 71

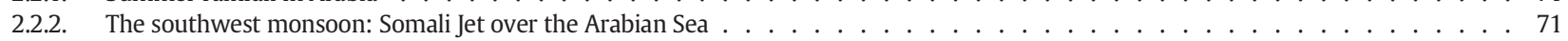

3. Lake versus marsh/wetland/discharge environments in arid lands . . . . . . . . . . . . . . . . . . . . . . . . . . . 71

\footnotetext{
* Corresponding author. Tel.: +972 2 6584210; fax: +972 25662581.

E-mail addresses: yehouda.enzel@mail.huji.ac.il (Y. Enzel), kushnir@ldeo.columbia.edu (Y. Kushnir), quadej@email.arizona.edu (J. Quade).
} 


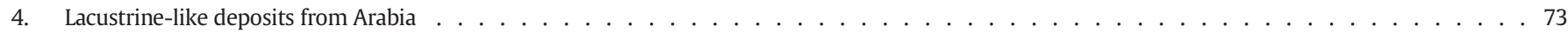

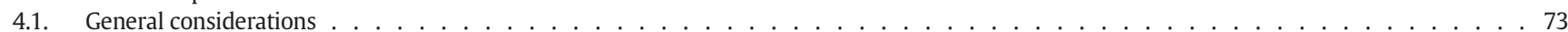

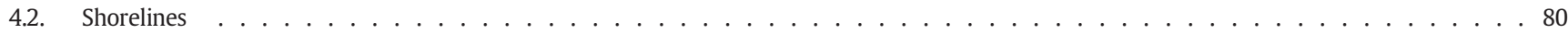

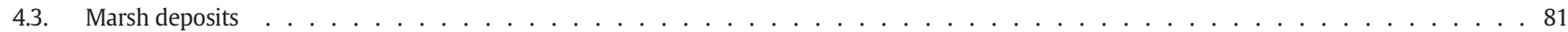

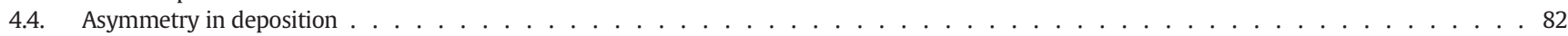

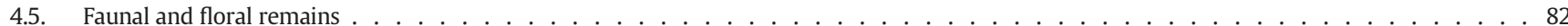

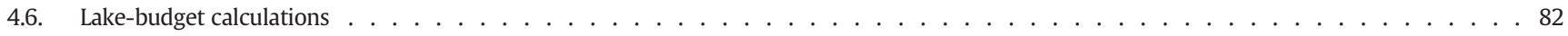

4.7. Summarizing lacustrine-like indicators . . . . . . . . . . . . . . . . . . . . . . . . . . . . . 8 83

5. Limited vegetation changes in Arabia . . . . . . . . . . . . . . . . . . . . . . . . . . . . . . . . 83

6. Rainfall shifts based on Speleothems ．.. . . . . . . . . . . . . . . . . . . . . . . . . . . . . . . . . . . . . . . . 83

7. Middle Holocene regional hydroclimatology and its implications . . . . . . . . . . . . . . . . . . . . . . . . . . . . . . . 84

7.1. Hydroclimatic pattern . . . . . . . . . . . . . . . . . . . . . . . . . . . . . . 84

7.2. Constraints on early to mid-Holocene climate . . . . . . . . . . . . . . . . . . . . . . . . . . . . . . . 85

7.3. Southwest monsoon winds and upwelling off-Oman assist keeping Arabia dry . . . . . . . . . . . . . . . . . . . . . . . 86

7.3.1. Upwelling off-southern Oman . . . . . . . . . . . . . . . . . . . . . . . . . . . . 86

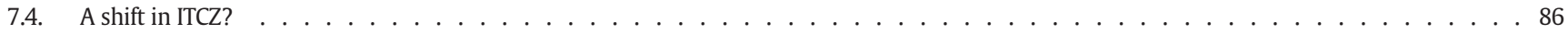

7.5. Implications to pre-Holocene interglacial climates of Arabia . . . . . . . . . . . . . . . . . . . . . . . . . . . . . 87

7.6. Implication for human-lacustrine association in Arabia . . . . . . . . . . . . . . . . . . . . . . . . . 87

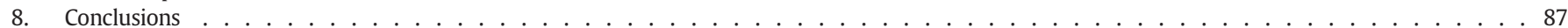

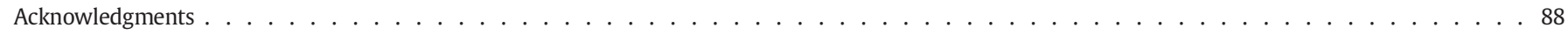

Appendix A. Supplementary data . . . . . . . . . . . . . . . . . . . . . . . . . . . . . 88

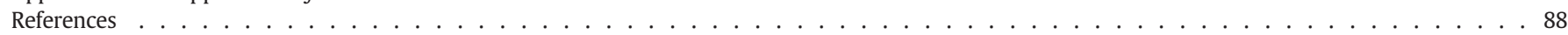

\section{Introduction}

Temporal patterns of Holocene lake-level status from Arabia to India (e.g., Street-Perrott and Harrison, 1985; Street-Perrott et al., 1989; Street-Perrott and Perrott, 1993; Kohfeld and Harrison, 2000; Braconnot et al., 2004) have led investigators to advocate large hydroclimatic changes during the early to middle Holocene in Arabia (e.g., Roberts and Wright, 1993). In a recent influential example, Wanner et al. (2008) mapped a $12^{\circ}-15^{\circ}$ northward shift in the middle Holocene monsoon rains over the Arabia-Near East-West Asia sector, based on paleoclimatic proxy-records including the lake status reconstructions for $6 \mathrm{ka}$. More conservative, but still substantial, estimates of northward displacement of the middle Holocene summer monsoon rainfall belt over Arabia and West Asia have also been proposed (e.g., Fleitmann et al., 2007; Fig. 11 in Junginger et al., 2014). Existing summaries of Holocene paleoclimates of the Arabian Sea and its African, Arabian, and Indian margins (e.g., Overpeck et al., 1996; Gasse, 2000; Hoelzmann et al., 2004; Prasad and Enzel, 2006; Staubwasser and Weiss, 2006; Fleitmann et al., 2007; Lézine et al., 2007, 2010; Wanner et al., 2008; Conroy and Overpeck, 2012) point primarily to the Indian summer monsoon (ISM) and to a lesser extent, to the African summer monsoons as explaining the amounts, seasonality and spatial distribution of past rainfall in Arabia.

The proposed northward expansion of summer rains across the Arabian Peninsula has been widely interpreted to represent a northward shift of the Inter-Tropical Convergence Zone (ITCZ) that has been proposed as a major driver of Holocene changes in the hydrology and vegetation in that area. This was postulated because of the proposed increased depth, area, or simply the presence of Arabian paleolakes. These lakes were taken as part of the evidence of high seasonal rainfall, which is needed to overcome the high rates of regional evaporation in the hyperarid Arabian Peninsula (currently is $>2200$ and as high as $\sim 3000 \mathrm{~mm} \mathrm{yr}^{-1}$, with pan or potential evaporation 3000$4000 \mathrm{~mm} \mathrm{yr}^{-1}$, see ElNesr et al., 2010; Sorman and Abdulrazzak, 1995). As several of these lake basins have no contributing watersheds, this implies a problematic assertion: the annual rainfall must have been at the magnitude of the above evaporation rates, unless these basins were primarily fed by regional groundwater flow or were only ephemeral; if such rainfall values are too high, then only ephemeral, shallow ponds could have formed.

\subsection{Structure of this reassessment}

In this paper we describe the present-day rainfall distribution in the Arabian Peninsula and the geographical and atmospheric mechanisms that control it. We then review the paleoenvironmental observations that underlie the proposed higher stands of the Arabian lakes. We use the information in the original reports and published research to distinguish between marshy or shallow water environments and open water bodies of lakes. This distinction is critical given the large moisture surplus necessary for the formation and maintenance of open lakes compared with a mosaic of wetland environments. Following this review we look at published pollen and speleothem data to gain a better picture of the region's paleoclimatology.

Finally, we propose explanations for the paleo-precipitation patterns emerging from the above lacustrine, speleothems, and pollen records, based on the modern atmospheric and ocean circulation in the northern Indian Ocean and its margins.

\section{Geographical and Meteorological constraints on Arabian rainfall}

\subsection{Annual and seasonal rainfall distribution}

The Arabian Peninsula is part of the Sahara-Arabian-Thar subtropical desert belt. As such its climate is dry, with most parts of the peninsula receiving $\leq 100 \mathrm{~mm} \mathrm{yr}^{-1}$ of rainfall (Fig. 1 ). The topography strongly affects the spatial pattern of the mean annual rainfall (Fig. 1) with only the elevated areas in the southwestern Peninsula experiencing $\geq \sim 500 \mathrm{~mm}$ annually (e.g., Subyani and Al-Dakheel, 2009) with highest points in Yemen receiving up to $800 \mathrm{~mm} \mathrm{yr}^{-1}$.

The seasonal distribution of rainfall (Fig. 2) indicates that most of the inner peninsula and the coastal strip along the Persian/Arabian Gulf and the Gulf of Oman coasts receive most of their rainfall in winter and spring. In contrast, the mountain ranges of southwestern Saudi Arabia and Yemen are dominated by summer rains (see also Almazroui, 2011; Almazroui et al., 2012, 2013).

\subsection{Synoptic climatology of Arabia rainfall}

We present several atmospheric circulation patterns that govern the winter and summer rainfall in different parts of the Arabian Peninsula. 
Their timing, magnitude and moisture sources are different indicating that a slight re-organization of these systems might create the basis for the change in rainfall patterns depicted by the paleoenvironmental records.

We have identified four rain-causing mechanisms that can affect the Arabian Peninsula during late fall, winter and early spring. a) Mediterranean cyclones that migrate into the Levant are dominant rainfall mechanisms in this region between October and May (Alpert et al., 2004; Almazroui et al., 2012). They bring little rain to Arabia, although they can become the dominant source of rainfall in the peninsula's east-northeast, relative to other seasons. b) The semipermanent low-pressure trough over the Red Sea, when combined with an eastward moving upper level trough can create local convective rainfall over the southern Levant, Sinai, and western and northern Arabian Peninsula and cause local flooding. c) The winter-spring tropical plumes (TPs, also can be considered as across-Sahara atmospheric rivers), which originate over the eastern tropical Atlantic or tropical Africa and move eastward across northern North Africa (Alpert et al., 2004; de Vries et al., 2013; Tubi and Dayan, 2014); occasionally, when combined with the subtropical jet positioned at a relatively southern latitude, these plumes can bring widespread, one to three days long, torrential rainfall and flooding into Arabia, Sinai, and southern Jordan and Israel (e.g., Ziv, 2001; Kahana et al., 2002; Barth and Steinkohl, 2004; Rubin et al., 2007; Tubi and Dayan, 2014); and d) northeast winter monsoon winds over the Gulf of Oman could have bring rain to the southeastern edges of Arabia.

\subsubsection{Summer rainfall in Arabia}

The broader regional pattern of monthly precipitation during summer (June-September, Fig. 3) indicates that southern Arabia falls between two regional monsoon systems. In the west, the African summer monsoon extends eastward along the sub-Saharan Sahel to the prominent local maximum over the Ethiopian Highlands and across the Red Sea into the mountains of southwestern Saudi Arabia and Yemen and westernmost Oman. Rainfall intensity in this part of the peninsula varies in phase with African summer monsoon intensity (Fig. 4). A band of rainfall extends farther east in Yemen into southern Oman, along the southern coastal topography (Fig. 1B).

The peninsula's interior is hyperarid, receiving $<80 \mathrm{~mm} \mathrm{yr}^{-1}$. The subsidence aloft induced by the heavy convection associated with the ISM (Rodwell and Hoskins, 1996), which is strongest over the East Mediterranean, extends over much of the Arabian Peninsula and possibly also brings northerly winds to southern Arabia and even to northern Arabian Sea region (Figs. 1 and 5). Moreover, the local high surface albedo together with the overlying dry air leads to a net loss of radiative energy, solar and infrared. The resulting radiative cooling of the atmosphere must be balanced by the adiabatic warming associated with large-scale subsidence, which further intensifies the drying of the desert (Charney, 1975). The moisture transport vectors (Fig. 3) and the rainfall minimum inland (Fig. 1b) suggest that the narrow zone of rainfall in coastal southern Arabia is associated with local orographic uplift (e.g., see left panels in Fig. 5 of Fleitmann et al., 2007). Very little moisture is transported inland across and over the coastal mountains. The low-level flow over the interior is mostly from the northwest (Fig. 2 in Ziv et al., 2004) and is dry and weak. Thus, in the interior of the Arabian Peninsula summer rainfall drops rapidly north of its Yemeni-Omani southern coast to $<10 \mathrm{~mm}$ for the entire summer season (Almazroui et al., 2012).

\subsubsection{The southwest monsoon: Somali Jet over the Arabian Sea}

The summertime southwesterly monsoon winds, which supply the moisture to the mountainous southernmost Arabian Peninsula, form a low-level jet, which peaks in intensity at altitudes of 1000-1800 m above sea level (Findlater, 1969a,b, 1971, 1977; Slingo et al., 2005). Referred to as the Somali or Findlater jet, these winds cross the equator in the trade wind belt north of Madagascar. The jet parallels the northeast
African coast, in part constrained by the high East African orography (Findlater, 1969a; see Fig. 5.31 in Krishnamurti et al., 2013) and forced by the northward pressure gradient. North of the equator, the lowlevel Somali jet is deflected by the Coriolis effect eastward from the East African highlands and across the Arabian Sea and heading directly towards India. Over the Arabian Sea the winds evaporate large amounts of moisture and eventually, over India and the Bay of Bengal, it feed the ISM rains (Findlater, 1971, 1977; Halpern and Woiceshyn, 2001; Slingo et al., 2005; Turner and Annamalai, 2012; Krishnamurti et al., 2013) with rainfall maxima where uplifted (Fig. 3).

\section{Lake versus marsh/wetland/discharge environments in arid lands}

Quantitative estimates of past changes in rainfall and temperature on the continents rely heavily on solving hydrological/energy budgets of past lakes (e.g., Mifflin and Wheat, 1979; Street-Perrott and Harrison, 1985; Benson, 1986; Benson and Paillet, 1989; Enzel, 1992). The main concept for these reconstructions is that at steady state in closed basin lakes, direct rainfall and runoff into a lake are balanced by evaporative losses (e.g., Street-Perrott and Harrison, 1985). The primary source of inflow for lakes in arid and semiarid basins is surface runoff (e.g., Mifflin and Wheat, 1979; Street-Perrott and Harrison, 1985; Enzel, 1992), as groundwater recharge and direct rainfall in these environments are usually low (e.g., Yechieli and Wood, 2002). The little recharge of groundwater when it occurs is more efficient in supplying water to terminal basins in arid regions as it is less susceptible to evaporation losses and leads to the formation of fresh to saline wetlands.

Clearly, an accurate reconstruction of a paleolake area is vital, since evaporative loss from a lake depends primarily on its surface area (e.g., Benson and Paillet, 1989). Surface area reconstruction is straightforward where paleolake shorelines are well preserved, but can be more difficult where shore deposits are old and/or local erosion rates are high. It is also important to clearly distinguish between lake and other lacustrine deposits in wet environments characterized by ground-water discharge such as springs, wet meadows, shallow marshes or discharging saline playas (Mifflin and Wheat, 1979; Rosen, 1991, 1994; Pigati et al., 2014).

In their seminal study of the hydrological basins in the Great BasinMojave deserts of the western United States, Mifflin and Wheat (1979) outlined for the first time a hydro-physiographic criterion for separating basins with evidence for paleolake existence from those without. Until this 1979 study, researchers had not been rigorous in distinguishing true lake deposits from the other types of deposits of similar appearance. This distinction is of the utmost importance given the very different paleohydrologic conditions required to support an open water body, even if it is a shallow lake, compared with other lacustrine environments. It requires much less water input into a drainage basin to maintain a shallow and patchy wetland or discharging playa, fed by groundwater that is mainly sheltered from direct evaporation than an open surface of a lake under $\sim 2 \mathrm{~m} \mathrm{yr}^{-1}$ of evaporation or even less.

Following studies using Mifflin and Wheat's (1979) approach, examined lacustrine-like sediments in lowland basins lacking shorelines, mainly in North and South America the distinction was possible. Researchers were able to define deposits of spring heads, wet meadows, shallow marshes and even shallow local pools, where these could be easily be mistaken for, and in some cases were actually reported as, lake deposits (Quade, 1986; Quade and Pratt, 1989; Quade et al., 1995, 1998, 2003; Rech et al., 2002, 2003; Pigati et al., 2009, 2011, 2014). Other examples of basins first defined as paleolakes and later shown as shallow wetlands are from northwest India (e.g., Enzel et al., 1999) and southern Jordan (Catlett et al., 2013, Catlett, 2014; Mischke et al., 2016). Table 1 summarizes the geomorphic, sedimentologic, and floral and faunal criteria that distinguish lake from wetland deposits (see also Pigati et al., 2014). This table guides us in re-interpreting the published information on the deposits in the basins of Arabia. 


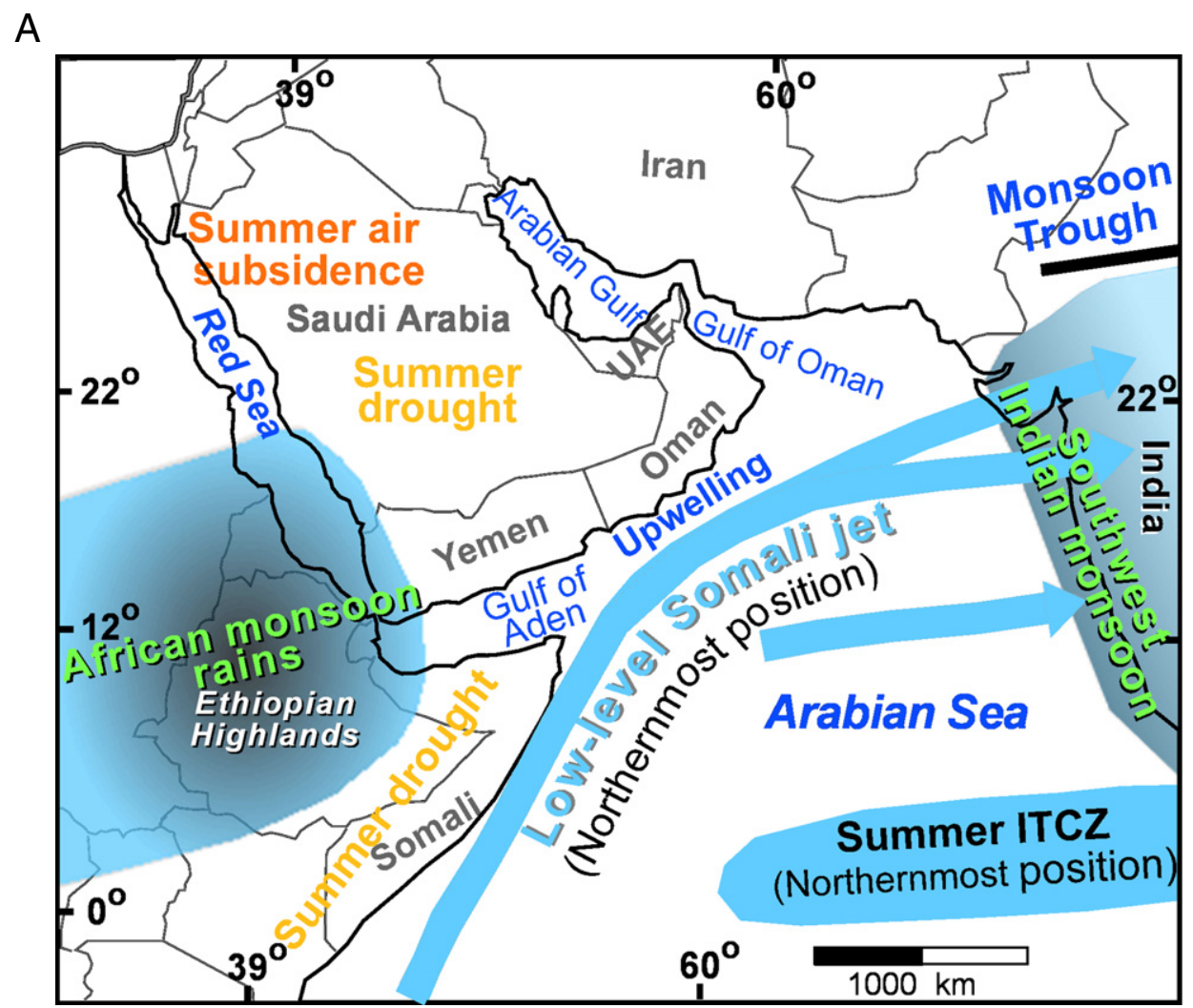

$\mathrm{B}$

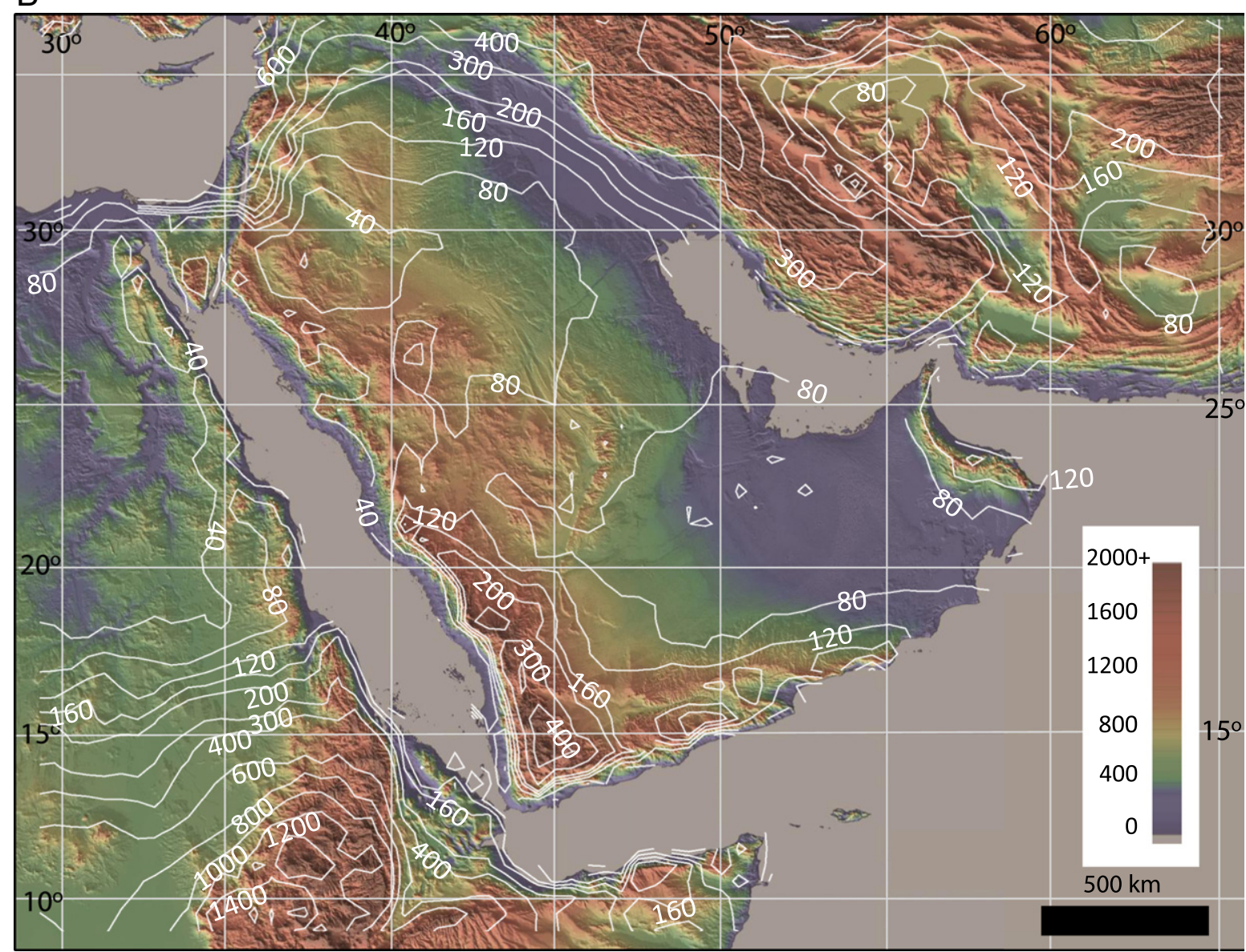



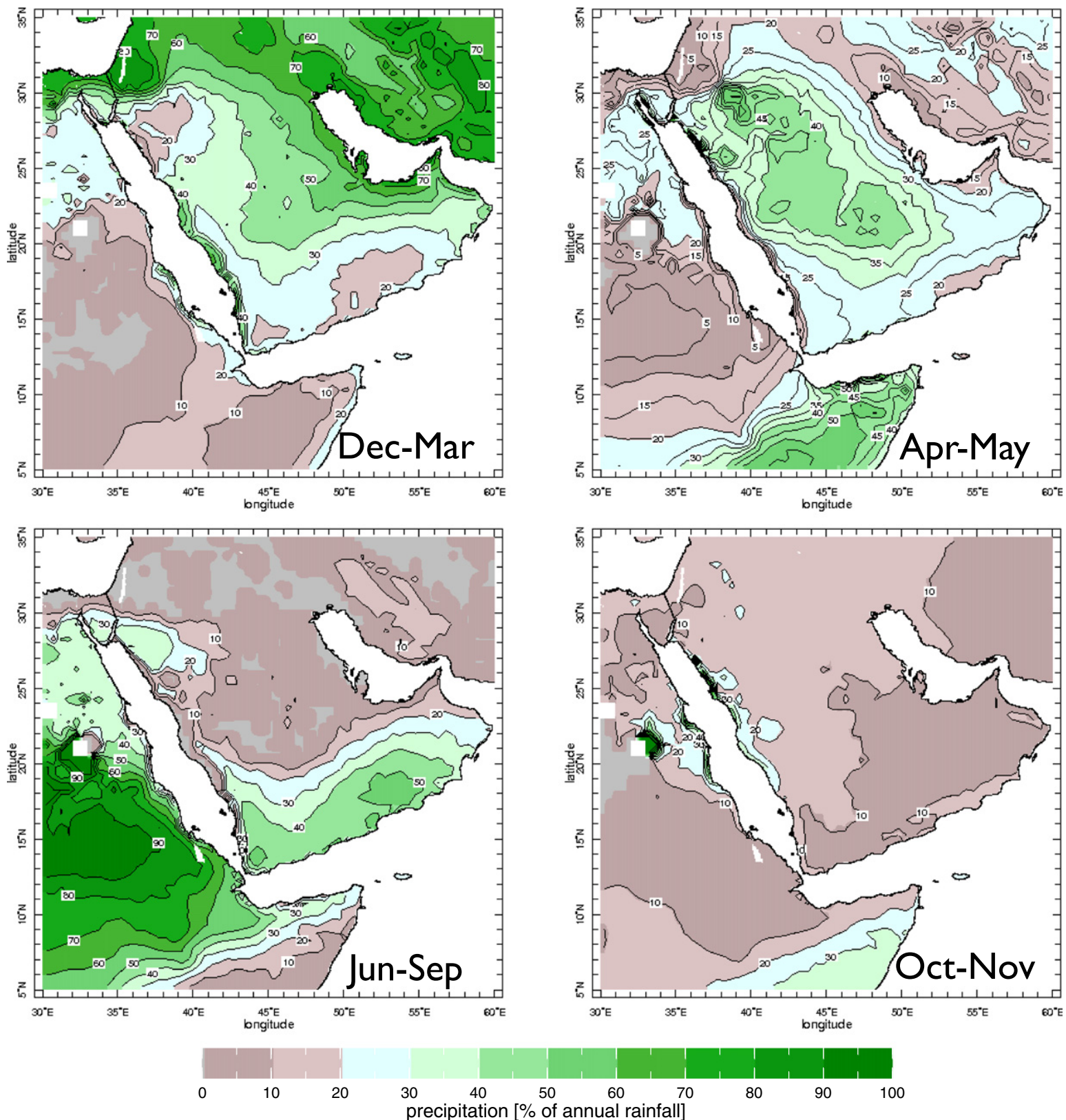

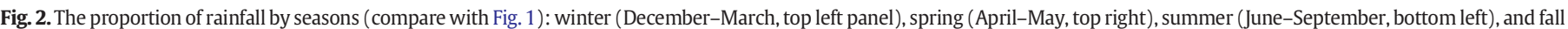

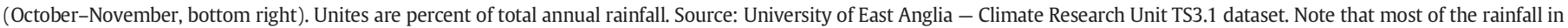

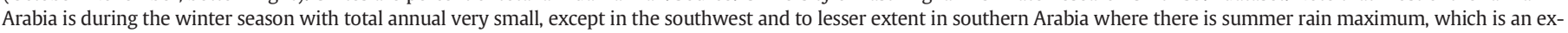

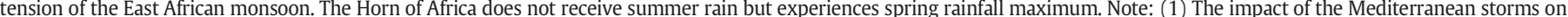

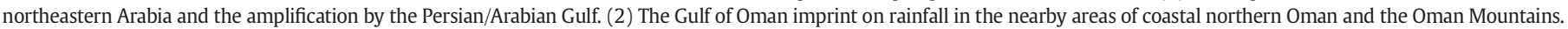

\section{Lacustrine-like deposits from Arabia}

\subsection{General considerations}

Based on the criteria shown in Table 1 we re-appraised the published sedimentary, floral and faunal characteristics and coexistence of these indicators in Holocene lowland deposits in Arabia (Table 2;
Fig. 6). We did not consider any pre-Holocene proposed lacustrinelike deposits such as Mudawwara in southernmost Jordan (Petit-Maire et al., 2010), which we think could also have benefited from additional scrutiny regarding its actual depositional environment, weak chronological controls, and identification and mapping of shoreline features as suggested by Catlett et al. (2013) and Rech et al. (2014). Recently, the Jubbah basin and other an-Nafud basins were proposed to

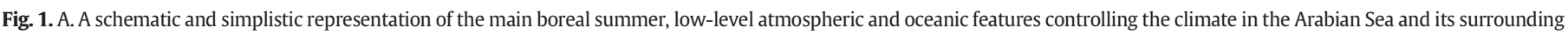

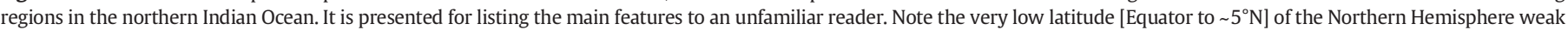

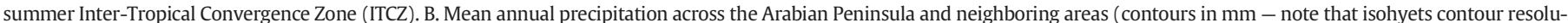

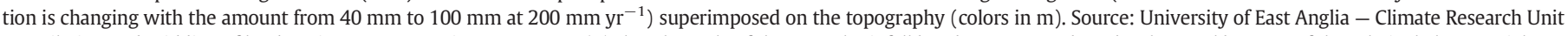

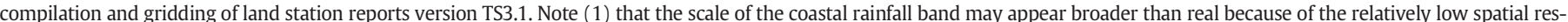

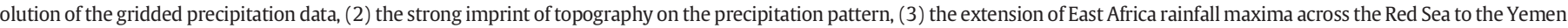

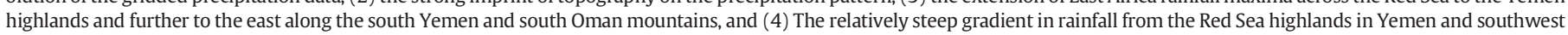
Saudi Arabia coast into the heart of Arabia to the east. 


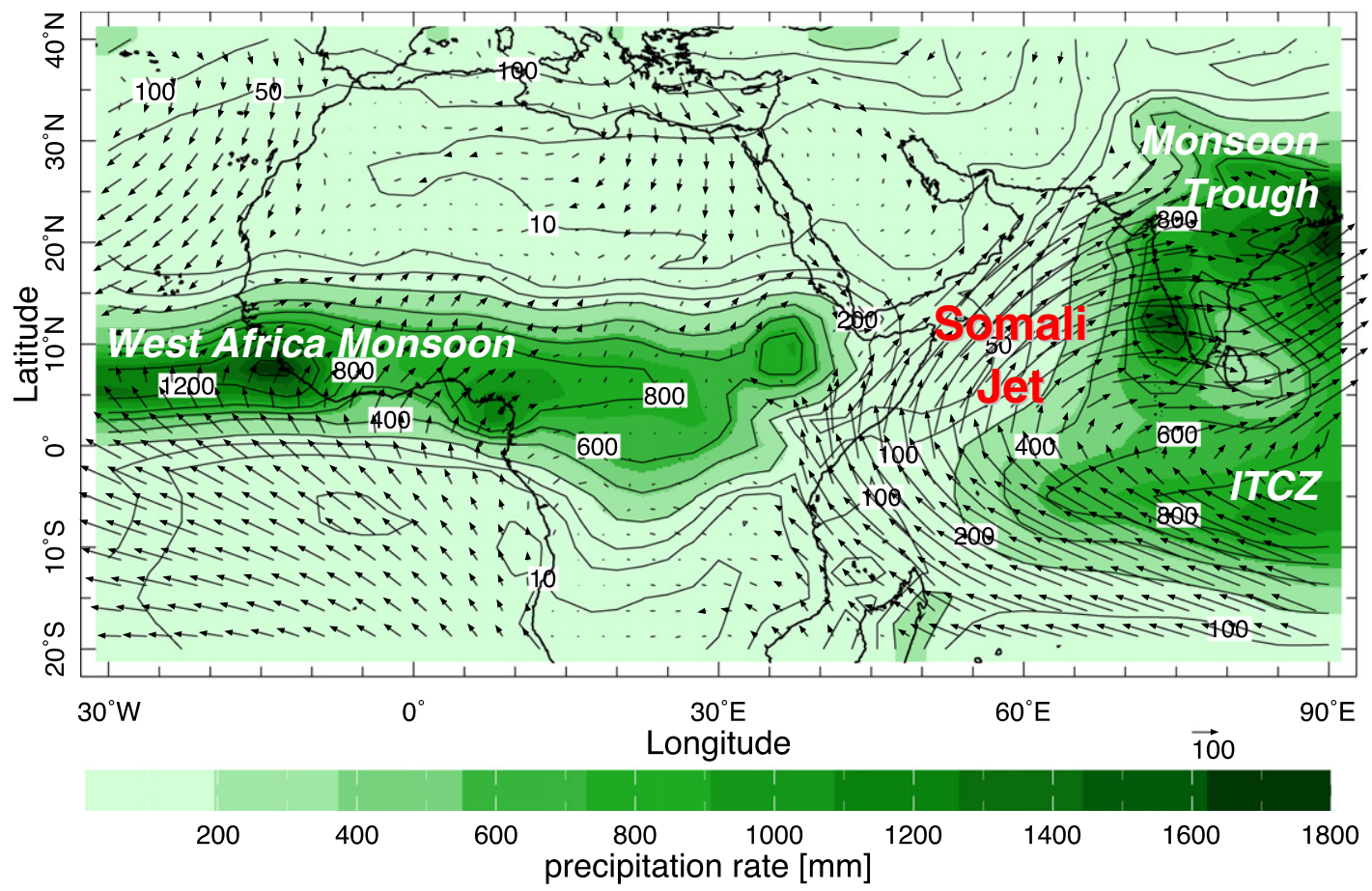

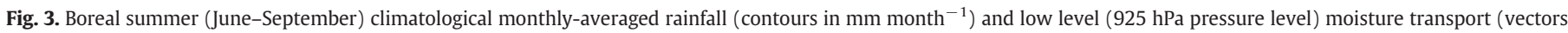

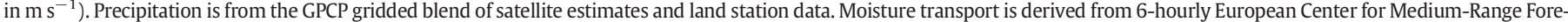

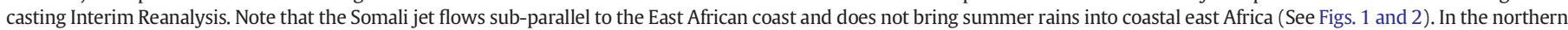

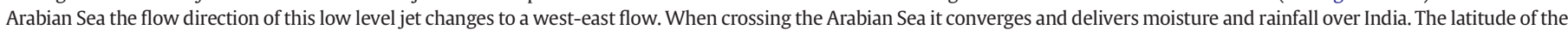

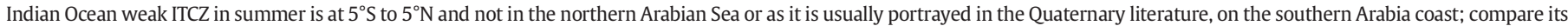
strength to the clear appearance in the east Atlantic Ocean at $5^{\circ}-10^{\circ} \mathrm{N}$.
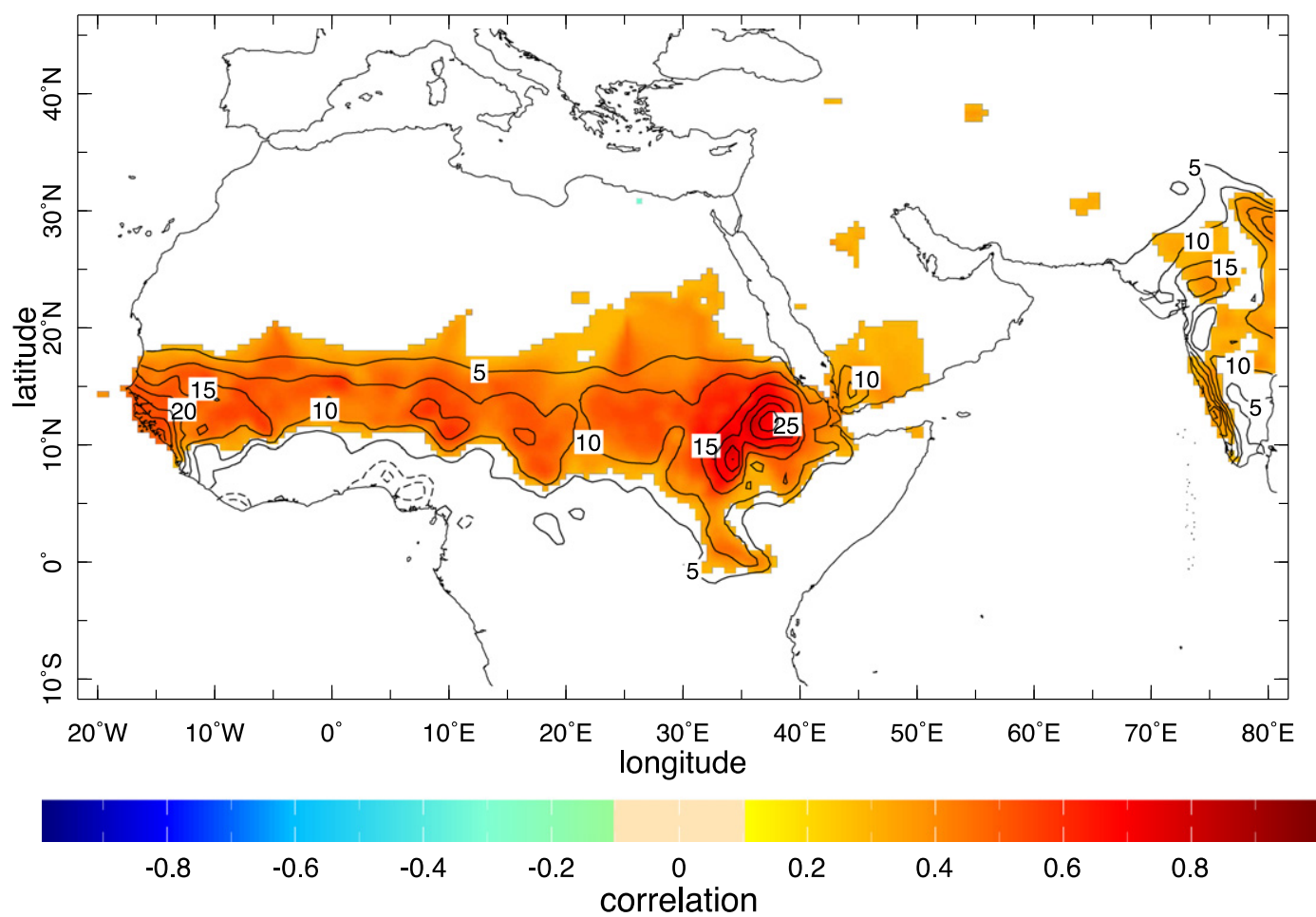

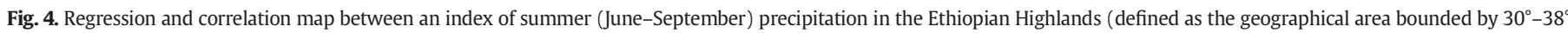

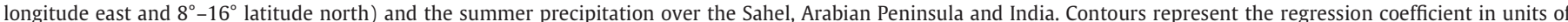

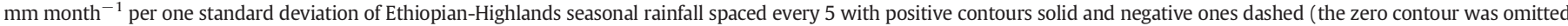

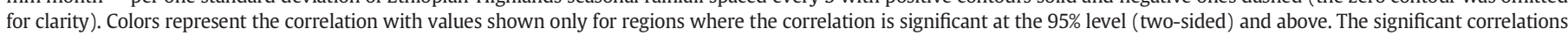
across Africa indicate the coherence of the entire African monsoon system, which extend into the southwestern Arabian Peninsula. 


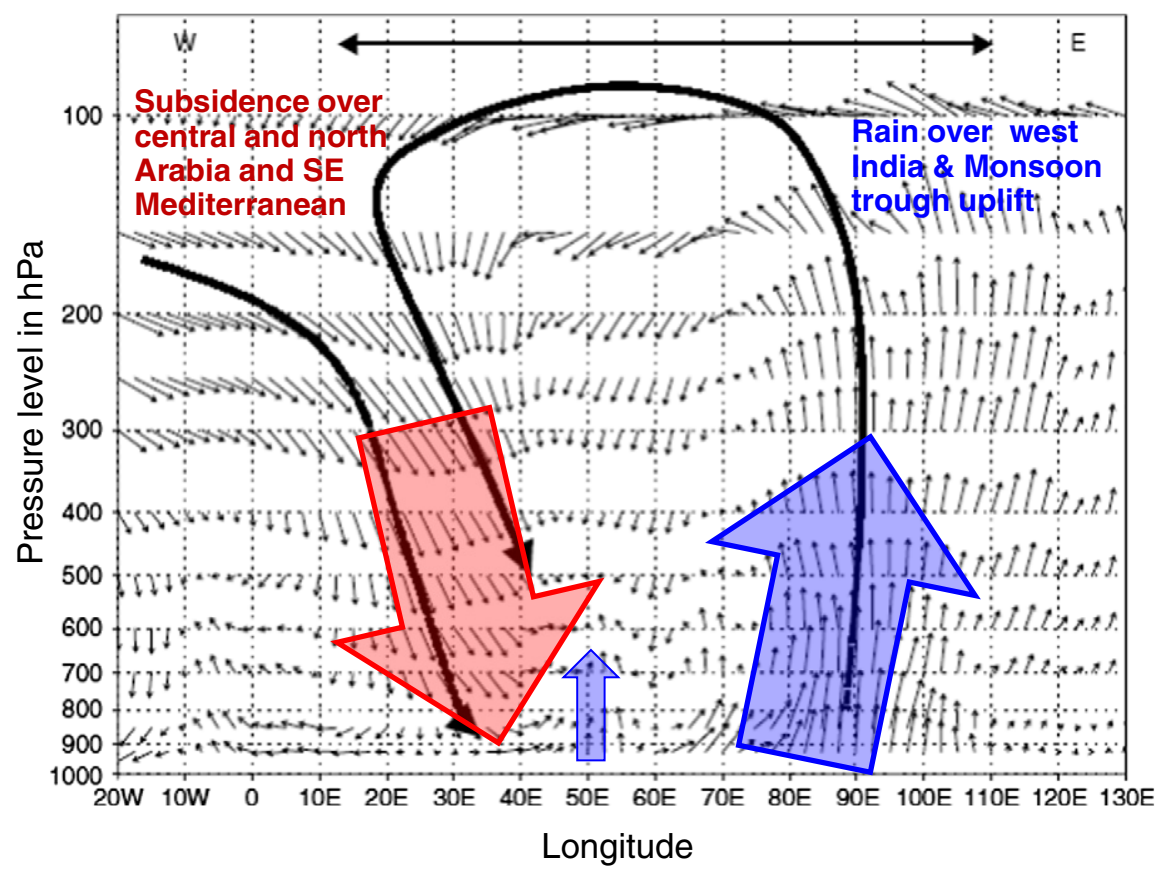

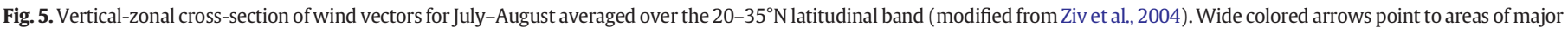
air subsidence and high pressure (red) and air uplift and low pressure (blue).

have been occupied by pre-Holocene lacustrine-like environments (e.g., Petraglia et al., 2011; Breeze et al., in press); they are not extensively discussed here either. However, before concluding that they were occupied by lakes rather than other types of depositional environment, they should also undergo (a) shoreline mapping beyond the remote sensing analyses of the basin flats and the determination of topographic closures (Petraglia et al., 2011; Breeze et al., in press) and (b) detailed sedimentologic and paleoenvironmental analyses listed in Table 1. We also chose to mention basins with reported Holocene ages even if later, these ages were disputed. The basins included here are:

- an-Nafud in northwest Arabia (Schulz and Whitney, 1986a) is included although Rosenberg et al. (2013) now suggest based on new OSL ages that these deposits may be actually pre-Holocene ( $>\sim 100 \mathrm{ka})$ deposits;

- Tayma, an oasis in NW Arabia with current groundwater at the surface (Engel et al., 2012);

- Mundafan in western Rub' al-Khali [(Figs. 6, 7 and McClure (1976, 1984) and Rosenberg et al. (2011)];

- The Oman Rub' al-Khali gypsum-crusted sabkhas (OR in Fig. 6; Figs. 8-11 in Matter et al., in press). Two such inter-dune depressions of early-middle Holocene age (11-5 ka) were studied (Matter et al., in press).

- The inter-dune Awafi beds at the base of the western Oman Mountains, UAE near the Arabian Gulf (Figs. 7, 8 and Parker et al., 2004);

- al-Hawa in Ramlat as-Sab'atayn (Figs. 6, 7 and e.g., Lézine et al., 2010);

- Interdune basins in Wahiba Sand field (Fig. 6 and Radies et al., 2004, 2005);

- Qa Jaran, Qa Zebl, Rada, Sada, and al-Adhla in the much wetter Yemen highlands (Fig. 6 and Davies, 2006; Lézine et al., 2010; Wilkinson, 2003);

- Maqta on top of southern Oman mountains (Fig. 6 and Fuchs and Buekert, 2008).

For each case we examine the presented evidence for paleoshorelines, aspects of the sedimentology and chronology, the types of aquatic and non-aquatic fauna, and in some cases also the implications of the $A_{w}$ values (Table 2) for paleohydrologic reconstruction.
The Holocene deposits at the Tayma oasis in northwest Arabia (Engel et al., 2012) illustrate the challenges in distinguishing groundwater discharge deposits from actual lake deposits. Tayma basin today hosts an artesian spring complex located on the western side of the small basin and apparently is recharged in the highlands to the west (Engel et al., 2012). A small salt pan occupies the bottom of the basin to the east, into which the spring and surrounding wadis discharged. The evidence for more abundant fresh to brackish water in the Tayma basin during the early Holocene (i.e., earlier than other sites) is unequivocal, given the distribution and faunal content of the deposits (Engel et al., 2012). The presence of laminated deposits with abundant fresh to brackish water fauna in the salt pan cores also points to permanent standing water at least thinly covering the salt pan, although the depth is unclear. More debatable, according to the criteria listed in Table 1, is the presence of a perennial (deeper than a few meters; see below) lake encompassing all these deposits. The fauna identified is in fact found in shallow to deep lakes today, but they are also known to exist in smaller permanent water bodies (e.g., the fresh-water gastropod Melanoides tuberculatus), such as springs, shallow wetlands, and small ponds.

The hyperarid basins of Mundafan and al-Hawa (Table 2) are fed by watersheds with wetter mountainous headwaters, currently receiving a maximum mean annual rainfall of $\sim 120 \mathrm{~mm}$ and $>200 \mathrm{~mm}$, respectively. Mundafan is in western Rub' al-Khali (McClure, 1976, 1984; Rosenberg et al., 2011) and is located at the end of a large drainage basin partially covered by sand (Figs. 5 and 6). Al-Hawa (Figs. 6 and 7) stands where the erg of Ramlat As-Sabatyan crosses the large Wadi al-Jawf-Wadi Hadramwat system in Yemen (extensive documentation in Lézine et al., 1998, 2007, 2010; Berger et al., 2012).

Farther east, in Oman's Rub' al-Khali there are numerous gypsumcrusted sabkhas (Fig. 9; and Fig. 5 of Sultan et al., 2008); two examples for such sabkhas are early-middle Holocene in age and probably represent a regional higher water table in the region in the past (Matter et al., in press). However, there are no indicators for past water bodies covering the sabkhas (Matter et al., in press). Sultan et al. (2008) suggest that the groundwater forming the sabkhas were fed from the west. However, they also considered recharge from elevated areas in the southern 
Table 1

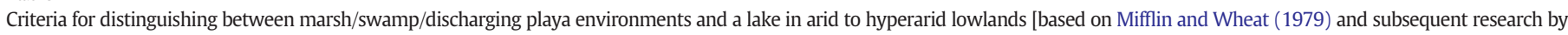

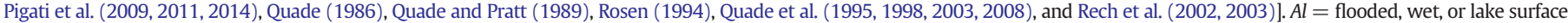

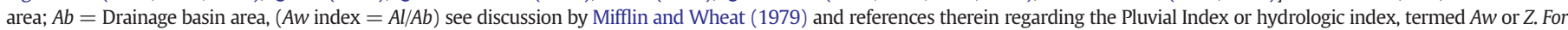

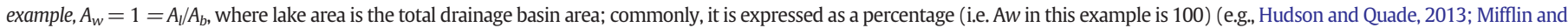
Wheat, 1979).

\begin{tabular}{|c|c|c|}
\hline & Marsh/swamp/discharge & Shallow/deep lake \\
\hline $\begin{array}{l}\text { Physiography and } \\
\text { landform } \\
\text { association }\end{array}$ & $\begin{array}{l}\text { Low elevation basins with local hydrographic closure and limited } \\
\text { contributing drainage area [i.e., high } A w \text { index]; These basins can be } \\
\text { inter-dunes or bordered by coalescent alluvial fans. }\end{array}$ & $\begin{array}{l}\text { Relatively large contributing drainage areas, higher elevation basins with } \\
\text { lower evaporation, hydrographically closed or with overflowing spillways } \\
\text { (e.g., low Aw index or low Pluvial Index, Mifflin and Wheat (1979). }\end{array}$ \\
\hline
\end{tabular}

Shore environment

a) Depositional

No shorelines per se, since wetlands, wet meadows, and springs are not high-energy (too shallow, not even minor waves); transition to margin at similar elevation.

b) Transition from basin's center to margin

c) Erosional

Small vertical difference between the flat basin floor and margins, basin margin deposits are usually flat, lateral transition to non-aquatic surroundings marked by browner color, increasingly un-bioturbated sand or silt, less nodular carbonate. Organic rich "black mats" locally common. Margin discharge zone sometimes surrounding the basin with accumulating fine grained deposits that can be rich in reeds and mistaken as beach deposit.

No erosional features by water body at margins. If groundwater has a role, their discharge zone is at the upstream margins and less than 1-2 $\mathrm{m}$ above basin floor and can be altered/eroded by aeolian deflation followed.

Basin sediments

a) Asymmetry

b) Delta

c) Bedding/lithology

Asymmetry in fine-grained deposits and thickness in the basin are pronounced with elevated deposition in the direction of incoming groundwater.

No delta deposits, alluvial fans may prograde into basin flats without the impact of standing water on their distribution.

Bioturbated green mudstone, marls, and locally extensive nodular carbonates and calcretes, sometimes full of root channels. Can be diatomaceaous where local waters are silica-rich. Evaporates exist only in true terminal saline wetlands where groundwater flow downstream is limited and evaporation controls outflow. Lamination can occur where algal mats are present or where seasonal variations in water table are associated with dust deposition.

Fauna Distinctive mix of terrestrial, semi-aquatic, and aquatic mollusks; and distinctive ostracodes (Quade et al., 1995, 1998). Fossil remains of large herbivores across the basin (i.e. not only at margins).

Flora Organic mats and algal mats with well-preserved sedges and grasses can be common. Reed vegetation is abundant as evident in organic-rich deposits, pollen, or root casts or all.
High-energy wave action produces beach berms and spits composed of reworked sediments of the surrounding landforms (e.g., dunes, alluvial fan) with distinct sedimentology of the well-sorted, cross-bedded sand and/or gravel, with noticeable unconformities. They are constructive, tens of centimeters to meters high landforms, cutting the preexisting topography. They occur even if lakes are only a few meters deep (e.g., Enzel et al., 1992). In carbonate-rich lakes these features are locally cemented by tufa into beach rock. Laterally continuous to semi-continuous.

Usually a large topographic difference between basin center and margins that present shoreline. The basin bottom is flat with or without a clear deeper point. No "black mats".

Wave-cut cliffs and/or polished surfaces in bedrock; minor gravel lags; tufa cemented where lake is carbonate rich.

Asymmetry in mostly related to clastic deposition at the entrance of the fluvial systems and evaporites are away from the fluvial system.

Fluvial systems end in delta deposits, especially where surface runoff is crucial for water budget. The delta top is usually at or near the level of shorelines. Bedded or laminated green to gray clay, silt, mudstone or marls but no major nodular carbonate. Diatomites where waters silica-rich. Evaporites associated with dry periods

Nearly all aquatic mollusks and distinctive ostracodes; terrestrial mollusk very rare. Larger herbivores at basin margins. edges of Arabia $\sim 200 \mathrm{~km}$ to the south. Fig. 9 indicates that headwaters of streams draining these mountains (with altitudes of $>1000 \mathrm{~m}$ ) northward to the Rub' al-Khali experience annual precipitation $>200 \mathrm{~mm} \mathrm{yr}^{-1}$. Furthermore, Fig. 5 of Sultan et al. (2008) points to the potential of these elevated southern mountains in explaining the isotopic composition of shallow groundwater in the southern sabkhas zone.

The Awafi inter-dune deposits (Table 2; Figs. 6-8 and Plate 4 in Parker and Goudie, 2008) are at a distal part of Wadi Naqal drainage basin $\left(\sim 75 \mathrm{~km}^{2}\right)$, where its alluvial fan (Al Farraj and Harvey, 2000, 2004) interacts with the United Arab Emirates (UAE) dune field (e.g., Goudie et al., 2000). The headwaters of Wadi Naqal are at altitudes $>1500 \mathrm{~m}$ at the western Oman Mountains, UAE, an area receiving only winter rains either by Mediterranean cyclones or by the northeast monsoon flowing over the Gulf of Oman. At the highest altitude of the watershed, the mean annual precipitation is $100-130 \mathrm{~mm} \mathrm{yr}^{-1}$ (Fig. 2 and Parker et al., 2004). Lower elevations in the watershed receive much less rainfall. Between the distal part of the alluvial fan and the Arabian Gulf (Figs. 6 and 8), the water table was near the surface before modern intensive ground-water pumping and, in places, even now. At a distance of $\sim 7 \mathrm{~km}$ from the Arabian Gulf, the Awafi inter-dune floor (Fig. 8) is
$<3-5 \mathrm{~m}$ above modern sea level. The top of the Holocene beds is $\sim 2 \mathrm{~m}$ below the inter-dune floor (Parker et al., 2004; i.e. 1-3 $\mathrm{m}$ above sea level at most) and therefore we suspect they were influenced by the low water table gradient $(\sim 0.5 \%$ ) to the gulf, especially during the sea level rising phase or even a slightly $(0.5-1 \mathrm{~m})$ higher sea level of the early Holocene. When the chronologies of Goudie et al. (2000) and Parker et al. (2004) are compared, it can be concluded that the dunes were established only shortly before the Awafi beds were deposited at the time of the early Holocene sea level rise. This indicates a genetic association between dunes, sea level rise, and rising water table to form the Awafi wetlands. Awafi is currently far from the influence of the monsoon summer precipitation and receives its rainfall mainly in the boreal winter and spring (Figs. 1 and 2).

The recently published Wahalah deposits from the United Arab Emirates (Fig. 6) are located $\sim 19 \mathrm{~km}$ southwest of the Awafi deposits (Preston et al., in press) and present similar settings. The current inter-dune basin floor is only 3-4 m meter above modern sea level (based on Google Earth) with the main deposit $\sim 1.5-2.2 \mathrm{~m}$ below basin floor (estimated from Preston et al., in press). Wahalah deposits are located $\sim 4.2 \mathrm{~km}$ from the Arabian Gulf indicating a very low gradient of $\sim 0.5 \%$ or less. Furthermore, an alternative to the C3-C4 


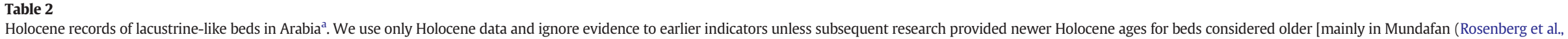
2011)]. Locations of basins are marked in Fig. 6 using the bold letters under basin name below.

\begin{tabular}{|c|c|c|c|c|c|c|c|}
\hline Basin name & Original report & Lat./long. & Indicators of environments & Shoreline features & Lake status $^{\mathrm{b}}$ & Vegetation (e.g., by) & Reinterpreted in this research \\
\hline $\begin{array}{l}\text { An-Nafud, NW Saudi } \\
\text { Arabia } \\
\text { N }\end{array}$ & $\begin{array}{l}\text { Thin lake beds } \\
\text { [Schulz and } \\
\text { Whitney } \\
\text { (1986a,b); } \\
\text { Whitney et al. } \\
\text { (1983); } \\
\text { Rosenberg } \\
\text { et al. (2013)] }\end{array}$ & $28^{\circ} 18^{\prime} / 40^{\circ} 56^{\prime}$ & $\begin{array}{l}\text { Cemented sand, desiccation } \\
\text { cracks, calcareous crusts, } \\
\text { laminated diatomites, fossiliferous } \\
\text { limestone, green claey silt, } \\
\text { burrows, numerous } \\
\text { roots/rhizome remains of } \\
\text { Phragmites and Typha and } \\
\text { Mollusks,"indicating swamps". }\end{array}$ & $\begin{array}{l}\text { Not reported by authors and not } \\
\text { observed in satellite images }\end{array}$ & $\begin{array}{l}\text { a) } 2 \text { (intermediate), in Roberts } \\
\text { and Wright (1993); (b) wetter } \\
\text { [Braconnot et al. (2004)]. } \\
\text { But "All lakes were essentially } \\
\text { of playa type" }\end{array}$ & $\begin{array}{l}\text { As today }{ }^{\mathrm{c}}+\text { long } \\
\text { distance transport } \\
\text { (Schulz and Whitney, } \\
\text { 1986a,b) }\end{array}$ & $\begin{array}{l}\text { Discharge deposits "at the } \\
\text { bottom of inter-dune } \\
\text { depressions" (Schulz and } \\
\text { Whitney, 1986a). Wetland } \\
\text { mosaic, with few extremely } \\
\text { shallow pools. }\end{array}$ \\
\hline $\begin{array}{l}\text { Nafud Asir /Urayk, } \\
\text { NW Saudi Arabia } \\
\text { N }\end{array}$ & $\begin{array}{l}\text { As above } \\
\text { w/one thick } \\
\text { sand-rich bed }\end{array}$ & $25^{\circ} 30^{\prime} / 44^{\circ} 30^{\prime}$ & Similar to the above & $\begin{array}{l}\text { Not reported by authors and not } \\
\text { observed in satellite images }\end{array}$ & As above & As above & As above \\
\hline $\begin{array}{l}\text { Tayma }^{\mathrm{e}} \\
\text { NW Arabia } \\
\text { T }\end{array}$ & $\begin{array}{l}\text { Engel et al. } \\
\text { (2012); Ginau } \\
\text { et al. (2012). }\end{array}$ & $\begin{array}{l}27^{\circ} 38^{\prime} \\
38^{\circ} 33^{\prime}\end{array}$ & $\begin{array}{l}\text { Layers of mud, laminated } \\
\text { aragonite, some ooids and graded } \\
\text { clastics (sand to mud), high } \\
\text { diversity faunal remains, } \\
\text { especially ostracodes (many } \\
\text { deformed); foraminifera; roots, } \\
\text { plant remains, evaporites (some } \\
\text { ringing the basin), gastropods. No } \\
\text { delta or alluvial fan, but with } \\
\text { pronounced asymmetry of } \\
\text { evaporates and other deposits } \\
\text { from south to north. }\end{array}$ & $\begin{array}{l}\text { Bioclastic remains several meters } \\
\text { above basin floor (Engel et al., } \\
\text { 2012, p. 137). Evidence for } \\
\text { permanent water above the } \\
\text { saltpan is laterally discontinuous } \\
\text { (or "disjunct", Engel et al., 2012, } \\
\text { p. 136). No shoreline is observed } \\
\text { in satellite images }\end{array}$ & Not in lake status database & No data & $\begin{array}{l}\text { Discharging shallow wetland, } \\
\text { with occasional standing water, } \\
\text { primarily fed by groundwater } \\
\text { and perhaps floods; rapid } \\
\text { fluctuations in and changing } \\
\text { salinities. }\end{array}$ \\
\hline $\begin{array}{l}\text { Mundafan', SW } \\
\text { Saudi Arabia, (Rub' } \\
\text { al-Khali Site 1) } \\
\text { M }\end{array}$ & $\begin{array}{l}\text { McClure } \\
(1984)\end{array}$ & $18^{\circ} 33^{\prime} / 45^{\circ} 19^{\prime}$ & $\begin{array}{l}\text { Mostly massive bedsg,h of sandy } \\
\text { marls, calcareous cemented } \\
\text { sandstones, algal "chara chalk" } \\
\text { (mats?), fossil reed (Phragmites) } \\
\text { and Typha, gastropods (mostly } \\
\text { Melanoides) and ostracodes, } \\
\text { foraminifera, diatoms, numerous } \\
\text { root and burrowing casts, } \\
\text { gypsum. Clays mostly } \\
\text { non-fossiliferous, Land snails } \\
\text { (summary in Table V-1, p. } 140 \text { of } \\
\text { McClure, 1984) }\end{array}$ & $\begin{array}{l}\text { None }^{\mathrm{i}} \\
\text { Not reported by authors and not } \\
\text { observed in satellite images }\end{array}$ & $\begin{array}{l}\text { (a) High, much wetter } \\
\text { (Roberts and Wright, 1993). } \\
\text { (b) Much wetter (Braconnot } \\
\text { et al., 2004) }\end{array}$ & $\begin{array}{l}\text { Grasses, mainly marsh } \\
\text { wet habitat. More } \\
\text { stable dunes. Slightly } \\
\text { wetter than northern } \\
\text { Rub' al-Khali. } \\
\text { (El-Moslimany, 1983, } \\
\text { 1990; McClure, 1984) }\end{array}$ & $\begin{array}{l}\text { Ponds, marshes, and possibly } \\
\text { discharge deposits from a } \\
\text { regional shallow alluvial aquifer } \\
\text { and floods related to the large } \\
\text { feeding drainage basin size } \\
\left(>2500 \mathrm{~km}^{2}\right) \text {. }\end{array}$ \\
\hline $\begin{array}{l}\text { Mundafan', SW } \\
\text { Saudi Arabia } \\
\text { M }\end{array}$ & $\begin{array}{l}\text { Rosenberg } \\
\text { et al. (2011) }\end{array}$ & As above & $\begin{array}{l}\text { Marl, sand, cemented sand, silt, } \\
\text { phytoclasts, burrows, root casts, } \\
\text { fossil reed, gastropods (numerous, } \\
\text { mainly Melanoides), and } \\
\text { ostracodes'. The foraminifera }^{1} \\
\text { identified characterize coastal } \\
\text { swamps, salt marshes, and } \\
\text { lagoons and in Mundafan brackish } \\
\text { water. Charophytes indicate } \\
\text { shallow depth.' }\end{array}$ & $\begin{array}{l}\text { None }^{\mathrm{k}} \text {. Not reported by authors } \\
\text { and not observed in satellite } \\
\text { images }\end{array}$ & As above & As above & $\begin{array}{l}\text { As above with indicators of very } \\
\text { sallow brackish water }\end{array}$ \\
\hline
\end{tabular}




\begin{tabular}{|c|c|c|c|c|c|c|c|}
\hline Basin name & Original report & Lat./long. & Indicators of environments & Shoreline features & Lake status $^{\mathrm{b}}$ & Vegetation (e.g., by) & Reinterpreted in this research \\
\hline $\begin{array}{l}\text { Al-Hawa, Yemen } \\
\text { H }\end{array}$ & $\begin{array}{l}\text { Lézine et al. } \\
(1998,2007 \text {, } \\
\text { 2010 })^{\mathrm{n}}\end{array}$ & $15^{\circ} 52^{\prime} / 46^{\circ}, 52^{\prime}$ & $\begin{array}{l}\text { Unconsolidated calcareous silts } \\
\text { biologic indicators and alternating } \\
\text { layers of consolidated calcareous } \\
\text { silts and diatomacaeous } \\
\text { calcareous silts, gastropod } \\
\text { (Melania tuberculata), Typha. }\end{array}$ & $?^{\circ}$ & Wetter (no category) & $\begin{array}{l}\text { Desert vegetation that } \\
\text { "do not indicate } \\
\text { changes" from modern } \\
\text { environment }^{\mathrm{p}}\end{array}$ & $\begin{array}{l}\text { Wetland w/occasional pools } \\
\text { and floods }{ }^{q} \text { and probably very } \\
\text { short lived shallow lakes. }\end{array}$ \\
\hline $\begin{array}{l}\text { Oman Rub' al-Khali. } \\
\text { South Oman sites } \\
\text { b18.2, b19.2 } \\
\text { OR }\end{array}$ & $\begin{array}{l}\text { Matter et al., in } \\
\text { press) }\end{array}$ & $19^{\circ} 16^{\prime} / 53^{\circ} 19^{\prime} ; 18^{\circ} 43^{\prime} / 53^{\circ} 11^{\prime}$ & $\begin{array}{l}\text { Gypsum and sand laminas, sand } \\
\text { with or w/out bedding, Twinned } \\
\text { and rosettes gypsum. Diagenesis } \\
\text { of gypsum. }\end{array}$ & None & $\begin{array}{l}\text { Not a lake, a capillary zone. } \\
\text { Higher regional groundwater. }\end{array}$ & Not reported & $\begin{array}{l}\text { Higher regional groundwater } \\
\text { than present. Recharge of } \\
\text { groundwater by streams } \\
\text { heading in the wetter Dhofar } \\
\text { Mts. of southern Oman (Fig. 9) }\end{array}$ \\
\hline $\begin{array}{l}\text { Awafi, UAE } \\
\text { A }\end{array}$ & $\begin{array}{l}\text { Parker et al. } \\
(2004)^{\mathrm{r}}\end{array}$ & $25^{\circ} 42^{\prime} / 57^{\circ} 55^{\prime}$ & $\begin{array}{l}\text { Marl, carbonates, organic, mottled } \\
\text { sand, phytolith (grasses), pollen } \\
\text { (Typha), algal material, carbon } \\
\text { isotopes on organic material }{ }^{\mathrm{s}} \text {, }\end{array}$ & $\begin{array}{l}\text { Not reported by authors and not } \\
\text { observed in satellite images or in } \\
\text { our field visit }\end{array}$ & Wetter (no category) & $\begin{array}{l}\text { "an expansion of } \\
\text { savannah grassland } \\
\text { w/woodland } \\
\text { elements" }\end{array}$ & $\begin{array}{l}\text { Wetland w/only occasional } \\
\text { standing very shallow water at } \\
\text { surface only } 1-2 \mathrm{~m} \text { above } \\
\text { Holocene sea level in } \\
\text { inter-dune area. }\end{array}$ \\
\hline $\begin{array}{l}\text { Wahalah, UAE } \\
\text { Wh }\end{array}$ & $\begin{array}{l}\text { Preston et al. } \\
\text { (in press) }\end{array}$ & $25^{\circ} 38^{\prime} / 55^{\circ} 47^{\prime}$ & $\begin{array}{l}\text { Carbonates }(<10 \%) \text { and other } \\
\text { minerals? within laminated sand } \\
\text { including ostracodes (Cyprideis } \\
\text { torosa), gastropods (Melanoides } \\
\text { tuberculata) at a specificunit. } \\
\text { Stable isotopes }{ }^{\mathrm{u}} \text {. }\end{array}$ & $\begin{array}{l}\text { Not reported. At this elevation } \\
\text { above sea level probably } \\
\text { impossible. }\end{array}$ & $\begin{array}{l}\text { Wetter. significant variations } \\
\text { in hydrology. }\end{array}$ & Not reported & $\begin{array}{l}\text { Coastal wetland with occasional } \\
\text { very shallow water at the } \\
\text { surface, related to water table; } \\
\text { only } 1-2 \mathrm{~m} \text { above Holocene sea } \\
\text { level in inter-dune area. } \delta^{13} C_{\text {org }} \\
\text { composition indicate very } \\
\text { shallow water }\end{array}$ \\
\hline $\begin{array}{l}\text { Qa Jaran, Yemen } \\
\text { QJ }\end{array}$ & Davies (2006) & $14^{\circ} 17^{\prime} / 44^{\circ} 31^{\prime}$ & $\begin{array}{l}\text { Lacustrine marl, clay, silt marsh } \\
\text { deposits, humic-rich buried soils, } \\
\text { carbonates, faint } \\
\text { micro-laminations, gastropods } \\
\text { (Melanoides tuberculata), root } \\
\text { casts. }\end{array}$ & $\begin{array}{l}\text { Not reported by authors and not } \\
\text { observed in satellite images }\end{array}$ & Wetter (no category) & No data & Marshy wetland \\
\hline $\begin{array}{l}\text { Qa Zebl, Yemen }{ }^{\mathrm{v}} \\
\text { QZ }\end{array}$ & Davies (2006) & $14^{\circ} 50^{\prime} / 44^{\circ} 38^{\prime}$ & $\begin{array}{l}\text { Lacustrine silt clay marl, } \\
\text { micro-laminations; green clayey } \\
\text { silt, carbonates. }\end{array}$ & $\begin{array}{l}\text { Not reported by authors and not } \\
\text { observed in satellite images }\end{array}$ & Wetter (no category) & No data & Marshy wetland \\
\hline $\begin{array}{l}\text { Rada, Yemen }{ }^{w} \\
\quad \text { Ra }\end{array}$ & $\begin{array}{l}\text { Lézine et al. } \\
\text { (2010) }\end{array}$ & $14^{\circ} 26^{\prime} / 44^{\circ} 49^{\prime}$ & $\begin{array}{l}\text { Diatomites indicating that the } \\
\text { lacustrine phase begun saline and } \\
\text { experienced short phase of } \\
\text { freshwater input. Silt layer, one } \\
\text { gastropod-rich layer. }\end{array}$ & $\begin{array}{l}\text { None }^{\mathrm{x}} \text { Not reported by authors } \\
\text { and not observed in satellite } \\
\text { images }\end{array}$ & $\begin{array}{l}\text { Wetter (no category); "the } \\
\text { water body remained } \\
\text { relatively shallow during the } \\
\text { whole lacustrine period" } \\
\text { Lézine et al. (2010) }\end{array}$ & No data & $\begin{array}{l}\text { Marshy salty to freshwater } \\
\text { wetland }\end{array}$ \\
\hline $\begin{array}{l}\text { Saada, Yemen }{ }^{\mathrm{y}} \\
\text { Sa }\end{array}$ & $\begin{array}{l}\text { Lézine et al. } \\
\text { (2010) }\end{array}$ & $17^{\circ} 00^{\prime} / 43^{\circ} 45^{\prime}$ & $\begin{array}{l}\text { Calcareous layer, fresh-water } \\
\text { gastropods. }\end{array}$ & $\begin{array}{l}\text { Not reported by authors and not } \\
\text { observed in satellite images }\end{array}$ & Slightly wetter & No data & Marsh \\
\hline $\begin{array}{l}\text { Al-Adhla, Yemen } \\
\text { Ad }\end{array}$ & $\begin{array}{l}\text { Wilkinson } \\
\text { (2003) }\end{array}$ & $14^{\circ} 31^{\prime} / 44^{\circ} 19^{\prime}$ & Clay w/freshwater mollusks & $\begin{array}{l}\text { Not reported by authors and not } \\
\text { observed in satellite images }\end{array}$ & $\begin{array}{l}\text { Wettest episode is reflected in } \\
\text { earliest Holocene "lake or } \\
\text { marsh" "pool or lake" } \\
\text { Wilkinson (2003) }\end{array}$ & No data & $\begin{array}{l}\text { Marsh or very shallow } \\
\text { lake/pools }\end{array}$ \\
\hline $\begin{array}{l}\text { Wahiba } \\
\text { Oman } \\
\text { Wa }\end{array}$ & $\begin{array}{l}\text { Radies et al. } \\
(2004,2005)\end{array}$ & $22^{\circ} 11^{\prime} / 58^{\circ} 489^{\prime}$ & $\begin{array}{l}\text { Gray patches in interdunes, } \\
0.6-1.3 \mathrm{~m} \text { thick. Bioturbated } \\
\text { fine-coarse silty sand. Gastropode } \\
\text { shells (dominated by Melanoides } \\
\text { tuberculata). Salinity tolerant } \\
\text { freshwater snails and ostracodes } \\
\text { and plant remains }{ }^{z}\end{array}$ & Not reported by authors & $\begin{array}{l}\text { Ancient "permanent lakes } \\
\text { with vegetated banks"aa } \\
\text { probably resulted from } \\
\text { groundwater rising into the } \\
\text { interdune surface } \\
\text { Short-lived but permanent } \\
\text { lakes }^{\mathrm{b}} \text {. Lacustrine interdune } \\
\text { deposits" }^{\mathrm{y}}\end{array}$ & No data & $\begin{array}{l}\text { High groundwater table } \\
\text { producing shallow short lived } \\
\text { lakes/marshes/pools in } \\
\text { interdunes. }\end{array}$ \\
\hline $\begin{array}{l}\text { Maqta } \\
\text { Oman } \\
\text { Ma }\end{array}$ & $\begin{array}{l}\text { Fuchs and } \\
\text { Buerkert } \\
\text { (2008) }\end{array}$ & $22^{\circ} 45^{\prime} / 59^{\circ} 00^{\prime}$ & $\begin{array}{l}\text { Playa-like silt and clay sediments } \\
\text { filling a small depression; this is a } \\
\text { small mountainous }(\sim 1000 \mathrm{~m} \\
\text { above sea level }) \text { depression; } \\
\text { increased sedimentation as } \\
\text { indicator of more inflows. }\end{array}$ & $\begin{array}{l}\text { Not reported by authors and does } \\
\text { not show in photograph (Fig. } 3 \text { of } \\
\text { Fuchs and Buerkert, 2008) }\end{array}$ & Playa-like sediments & No data & Occasionally flooded playa. \\
\hline
\end{tabular}


Notes to Table 2

a We use only Holocene data.

b Lake level status: Low (1); intermediate (2, corresponding to slightly wetter); high (3, corresponding to much wetter). e.g., Braconnot et al. (2007a,b), Roberts and Wright (1993), Street-Perrott and Harrison (1985). More information at http://www.bridge.bris.ac.uk/projects/GLSDB.

c “... didn't exceed ... semidesert comparable to the modern plant cover" Schulz and Whitney (1986a,b).

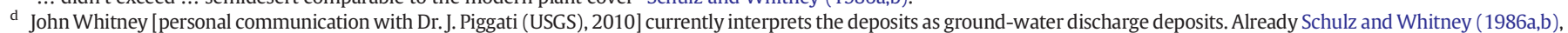

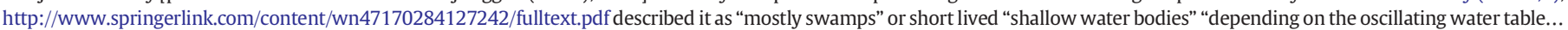

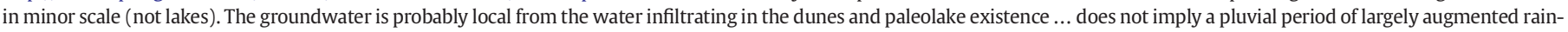

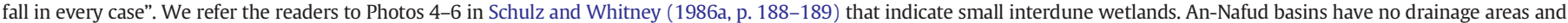

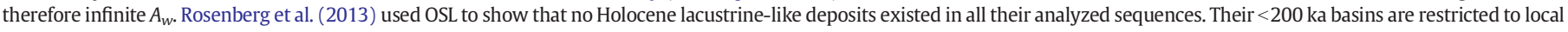
interdune depressions.

e This is the only research of any lacustrine-like deposit that also suggests evidence of possible shoreline several meters above current playa floor (Engel et al., 2012; Ginau et al., 2012). See

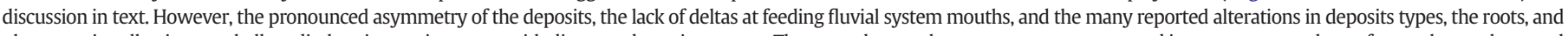

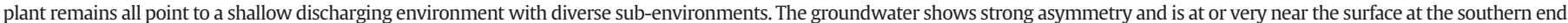

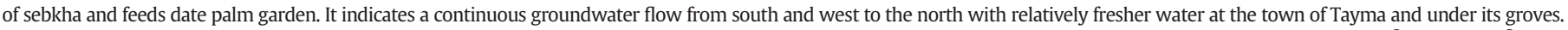
Tayma's $A_{w}$ value is $1-3 \%$ [this large range is due to the different $A_{d}$ reported by Ginau et al. (2012), Engel et al. (2012), and Wellbrock and Grottker (2010) of 1950 km ${ }^{2}$ and 660 km²].

f McClure (1984, p. 200): "Rub' El Khali lakes were due to temporarily perched, ephemeral and local occurrences of rainfall saturated zones". McClure (1984) advocated local

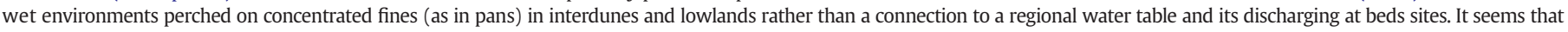

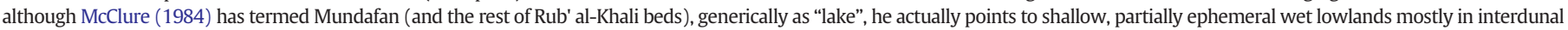

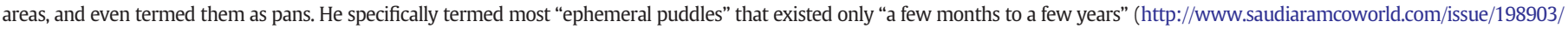
lakes.of.the.rub.al-khali.htm). $A_{w}=0.5 \%-0.6 \%$.

g Mundafan beds are thin beds distributed across the Mundafan basin and were first described and dated by radiocarbon by McClure (1976, 1984). Recently, Rosenberg et al. (2011)

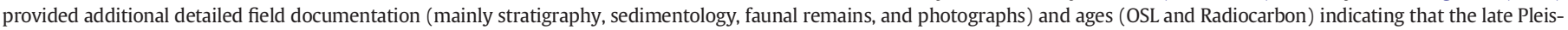

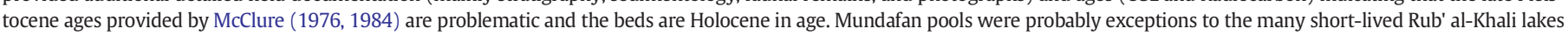

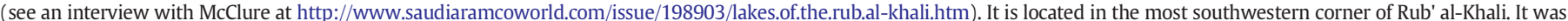

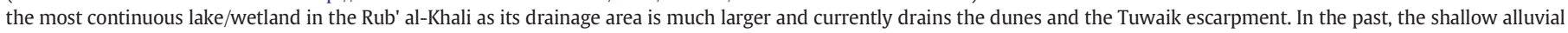

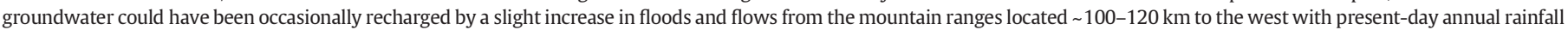
120-150 mm, a process common in allochtonous arid drainage basins (e.g., Enzel and Wells, 1997).

h e.g., Plate 2 in McClure (1984)

${ }^{i}$ Although Roberts and Wright (1993, p. 208) report a lake level curve for Mundafan and cited McClure (1984) as the source of data, there is no such curve in his writings. The various

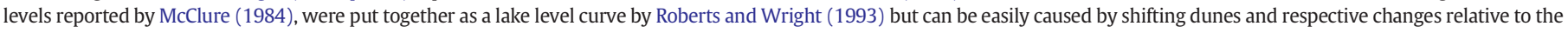
lowest points in basin (e.g., McClure, 1984, p. 82).

${ }^{j}$ Rosenberg et al. (2011) present photographs of the deposits (see their GSA Data Repository 2011333). These photographs indicate that a wetter environment existed at Mundafan lowland, but not a true lake; these are discharge marshy deposits.

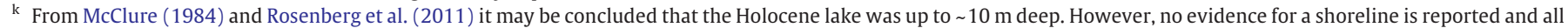
the stratigraphic data from the lowland are within 2-3 m, potentially indicating very shallow water body/bodies.

${ }^{1}$ Ostracode assemblage indicates perennial, but shallow water body and the foraminifera (a) tolerate a wide range of salinity, (b) characterize marine coastal swamps, salt marshes, and

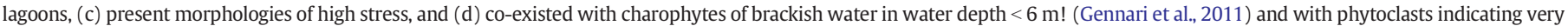

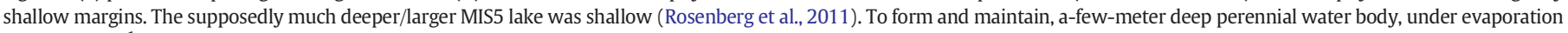

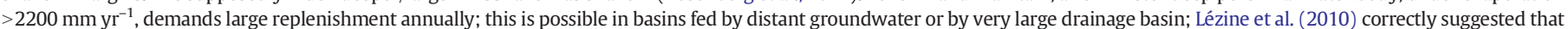

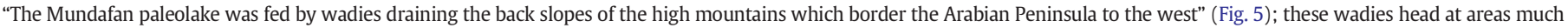
wetter $\left(120-150 \mathrm{~mm} \mathrm{yr}^{-1}\right)$ than Mundafan.

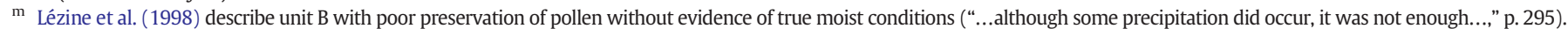

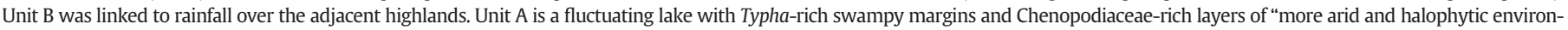
ment"; i.e. the water is relatively shallow with evaporation at is margins.

${ }^{\mathrm{n}}$ Lézine et al. $(1998,2007)$ reported two different pits dug into the al-Hawa bed ( $2 \mathrm{~m}$ and $7.45 \mathrm{~m}$, respectively) and two sets of radiocarbon ages.

o No shoreline was reported for this basin. We identified 2-3 undated elongated geomorphic features at $15^{\circ} 52^{\prime} 50^{\prime \prime} \mathrm{N} 46^{\circ} 54^{\prime} 30^{\prime \prime} \mathrm{E}$, approximately $350 \mathrm{~m}$ to the east and subdued features

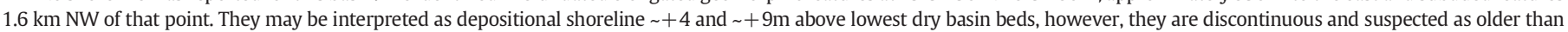
Holocene features.

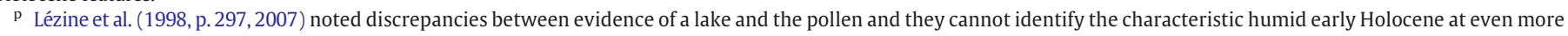

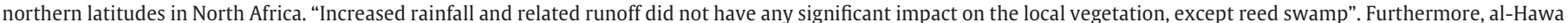

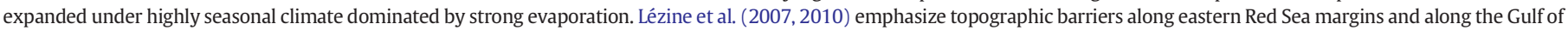

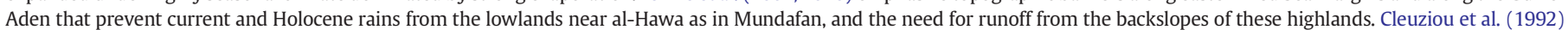

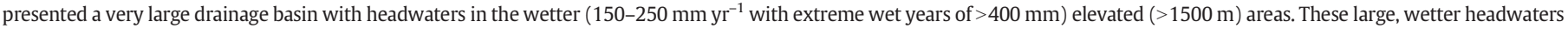

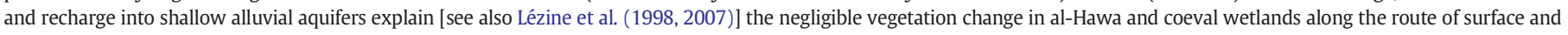
subsurface flows.

q Based on photographs presented in Lézine et al. (2007, p. 237) and comparing them with other wetland/discharge deposits.

r Small $\left(<2 \mathrm{~km}^{2}\right)$ dry lake beds as part of a few such dry basins at similar altitude at the north-easternmost Rub' al-Khali. Note that we used the longitude provided in Fig. 5 of Parker et al.

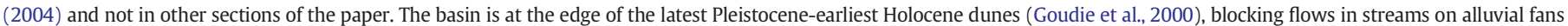

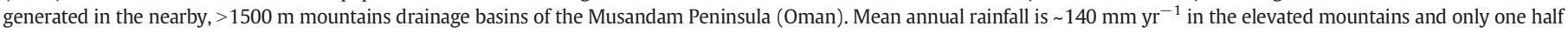

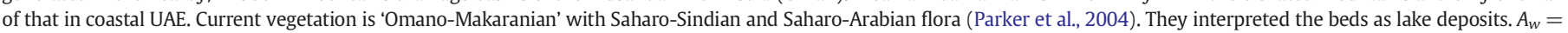
$0.1 \%-0.2 \%$ if the contributing basin is considered as Wadi Naql, the one forming the alluvial fan; otherwise, $A_{w}$ is very large.

${ }^{s}$ Interpreting the $\delta^{13} C_{o m}$ as representing variations in C3-C4 vegetation is problematic in the presence of algae mats (Parker et al., 2004, p. 673). In such a case $\delta^{13} C_{\text {om }}$ may represent

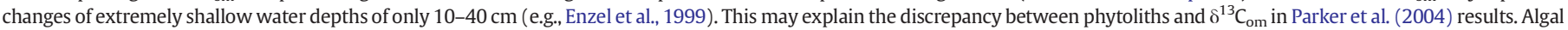
mats produce laminated deposits, easily mistaken for lacustrine laminations (e.g., Enzel et al., 1999).

${ }^{t}$ The Awafi beds (and nearby small basins) are only a few meters above current sea level, at the toe of active alluvial fan $\left(>10 \mathrm{~km}^{2}\right)$ of much larger $\left(<100 \mathrm{~km}{ }^{2}\right)$ drainage basins heading

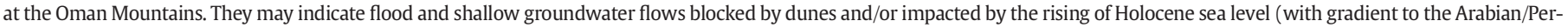
sian Gulf even lower than today (Fig. 8) in the middle Holocene as sea level was slightly higher) and the associated rise in water table.

u See text (Section 4.2).

$\checkmark$ Davies (2006) reported two lacustrine basins $\left(14^{\circ} 35^{\prime} \mathrm{N}, 44^{\circ} 31^{\prime} \mathrm{E}\right.$ and $\left.14^{\circ} 17^{\prime} \mathrm{N}, 44^{\circ} 35^{\prime} \mathrm{E}\right)$ at Dhamar highlands, central Yemen, not far from the headwaters of the basin discussed by

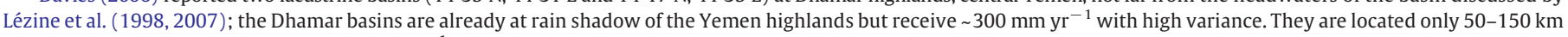

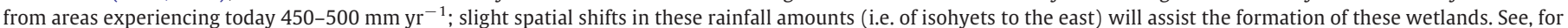
example, Cleuziou et al. (1992) who suggested $\sim 400 \mathrm{~mm} \mathrm{yr}^{-1}$ during modern exceptionally wet years in the headwater region.

$\mathrm{w}$ This very high elevation (> $2000 \mathrm{~m}$ ) basin (Lézine et al., 2010) is located at area receiving mean annual precipitation of $250-300 \mathrm{~mm}^{-1}$.

${ }^{x}$ No shoreline was reported but a faint feature $\sim 1-2 \mathrm{~m}$ above basin floor observed in satellite images may be interpreted as a shoreline but equally as zone of discharging water. In any case, it is discontinuous and vague, and therefore probably not of Holocene age.

$\mathrm{y} \sim 200 \mathrm{~mm} \mathrm{yr}^{-1}$.

z Radies et al. (2005). Very large $A_{w}$.

aa Radies et al. (2004, p. 1373) summarize also earlier data.

bb In addition, these authors point to the lack of soil development as indicator of only subtle impact of early Holocene climate on the Wahiba dunes. 


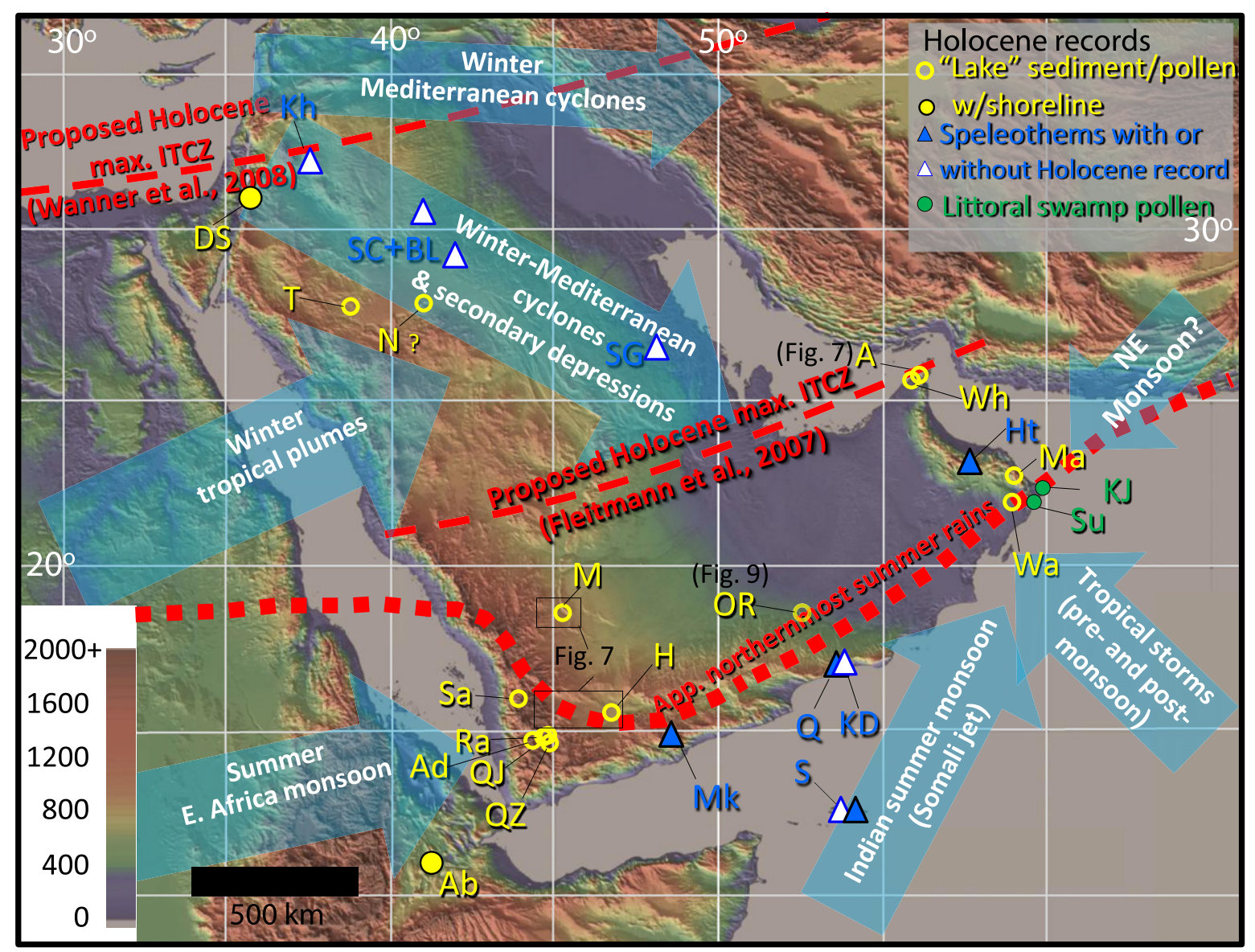

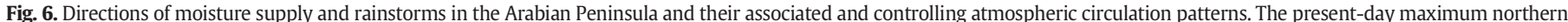

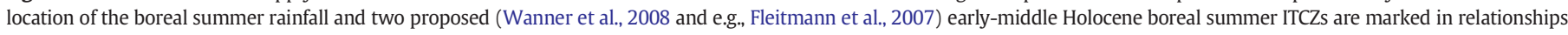

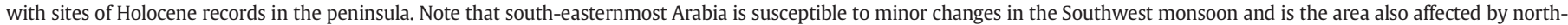

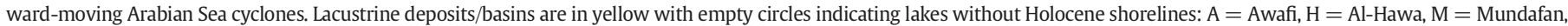

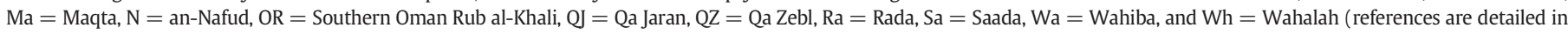

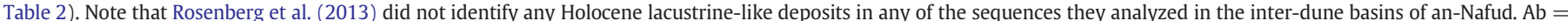

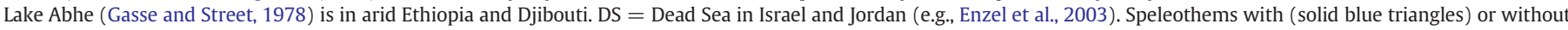

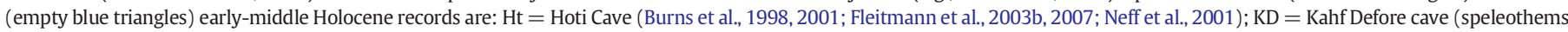

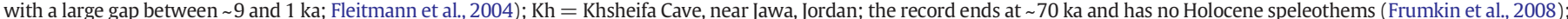

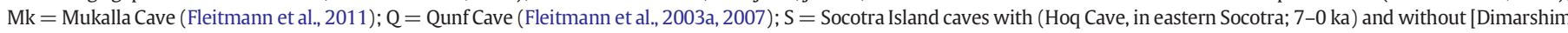

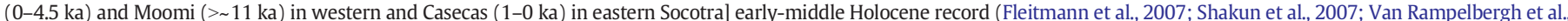

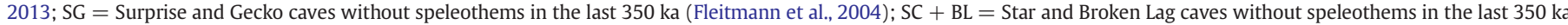
(Fleitmann et al., 2004). KJ = Kwar al Jaramah and Su = Suwayh are pollen records from Lézine (2009) and Lézine et al. (2010).

interpretation of the measured $\delta^{13}$ Corg along this section, can use the Baertschi effect (and not the change in C3-C4) that is best applicable in such environments where algal mats produce organic matter (e.g., Enzel et al., 1999 and references therein). If used, the $\delta^{13}$ Corg results (Preston et al., in press) indicate that the water depth throughout the wetland existence was always extremely shallow, probably not more that $10-20 \mathrm{~cm}$. All these evidences indicate that rather than being interpreted as a lake (Preston et al., in press), these deposits probably indicate coastal inter-dune sabkha with the characteristic sandy laminas that are largely impacted by sea level and groundwater. Furthermore, the similar chronologies of Awafi and Wahalah deposits ( 8.5-6 ka) are both associated with maximum sea level.

The late Pleistocene and Holocene inter-dune basins in an-Nafud in northwestern Arabia (Schulz and Whitney, 1986a; Rosenberg et al., 2013) have no drainage basin and may not contain Holocene deposits as suggested earlier (Table 2). Al-Adhla, Qa Jaran, Qa Zebl, Rada, and Sada are located in the much wetter Yemen highlands (Wilkinson, 2003; Davies, 2006; Lézine et al., 2010) and are impacted by the North African summer rains. However, even in this wetter environment the basins were occupied by wetlands and not by lakes (Table 2), but this may be the result of basin physiography and surface and subsurface outflow from these wetlands.

\subsection{Shorelines}

Among all the distinguishing features of paleolakes, perhaps most important is the presence of fossil shorelines. Distinct shoreline composed of gravel or sandy berms with $>0.5 \mathrm{~m}$ relief can form around lakes in little as a single season to a few decades, depending on local conditions (Hunter et al., 1990; Enzel, 1992; Enzel et al., 1992; Thompson and Baedke, 1995; Adams and Wesnousky, 1998; Bartov et al., 2006). By contrast, shorelines are not found around springs nor shallow ground-water fed wetlands $<\sim 3 \mathrm{~m}$ deep, nor in ephemeral (usually seasonal) wet playas, pans, or sabkhas. Late-Quaternary-age shorelines indicative of stable paleolakes should be well preserved in the Arabian Peninsula, given its extreme aridity and generally modest relief. Paleoshorelines of late Quaternary (25-7 ka) age are amply preserved, for example, in the Great Basin of the western USA (Mifflin and Wheat, 1979), central Tibet (Hudson and Quade, 2013), Patagonian South America (Stine and Stine, 1990; Anselmatti et al., 2006), and sub- 


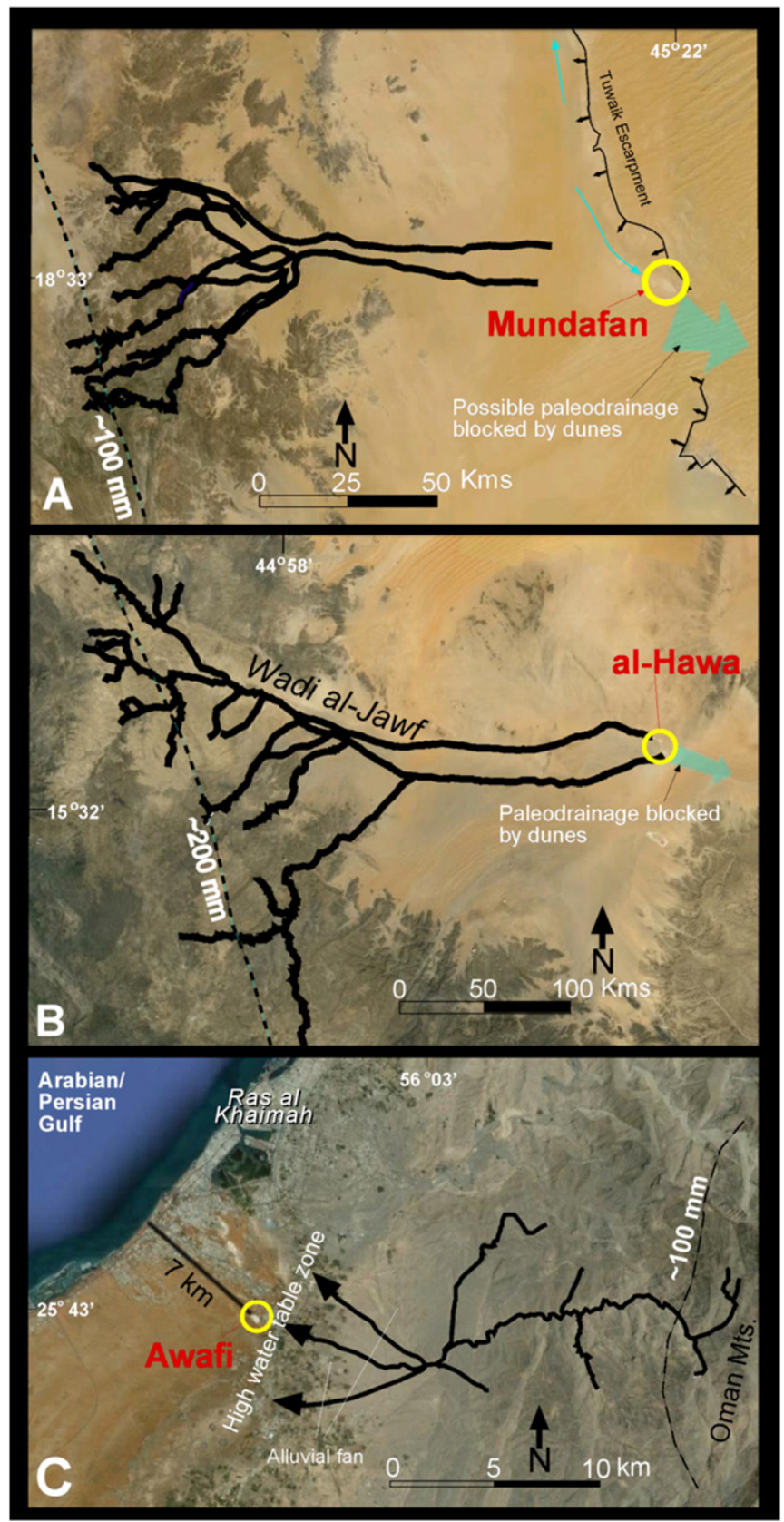

Fig. 7. The drainage basins of (A) Mundafan, (B) Al-Hawa, and (C) Awafi and the current isohyets of 100 and $200 \mathrm{~mm}$ in their respective drainage basins. Note the different scales for these basins. See Fig. 6 for location. Their drainage areas are $\sim 2500-3000 \mathrm{~km}^{2}$ and $13,000-14,000 \mathrm{~km}^{2}$, and $\sim 80-100 \mathrm{~km}^{2}$, respectively according to topography, but portions of these drainage basins may not drain directly to the specific basin. Additional rains in southwestern Arabia highlands will shift these $200 \mathrm{~mm}$ and $100 \mathrm{~mm}$ isohyets to the east to cover the respective drainage basins of al-Hawa and Mundafan by more rains. Such past shifts by as little as $100-200 \mathrm{~km}$ will dramatically increase the rainfall, recharge of shallow alluvial aquifer and flows. The line (marked as "7 km") in C tracks the location of the schematic cross section in Fig. 8.

Saharan Africa (Burrough and Thomas, 2009). These regions are wetter than peninsular Arabia today, and the paleolakes often reside in highrelief structural basins, leading to widespread erosion of shorelines. Despite this, preservation of $>50 \%$ of $\sim 17 \mathrm{ka}$ shoreline berms is quite typical in the Great Basin (Mifflin and Wheat, 1979), even in the geomorphologically very active Death Valley system. Were paleolakes present, we would therefore expect widespread preservation of late Quaternary (25-6 ka) paleoshorelines in peninsular Arabia, except where locally buried by younger dunes. However, among all these lacustrine-like deposits listed previously, there is no reporting of either depositional or erosional paleoshoreline even a few meters higher than the deposits and therefore no clear evidence of lake level. This matches our own inspection of satellite images of all these basins. For example, Engel et al. (2012) describe in Tayma the abundant fine-grained deposits and fresh-water fauna up to an altitude of $811.5 \mathrm{~m}$, i.e. $\sim 11 \mathrm{~m}$ above equivalent-age layers under the modern saltpan. They conclude that at 9-10 ka, thousands of years earlier than most other lacustrine evidence from Arabia, a $\sim 18-\mathrm{km}^{2}, \sim 13-\mathrm{m}$ deep lake occupied the Tayma basin. The observations from satellite images suggest to us that Tayma was probably not occupied by the deep lake envisioned by Engel et al. (2012). Field-based geomorphic mapping of shorelines was not provided for Tayma (or in this matter, for any proposed Holocene or earlier lake in Arabia!). The basin conspicuously lacks evidence of shorelines at the $811.5 \mathrm{~m}$ or for that matter at any elevation. $\mathrm{A} \geq 13 \mathrm{~m}$ deep lake during the early-Holocene would have left conspicuous and nearly continuous paleoshorelines around the Tayma basin. Permanent water above the Tayma saltpan is laterally discontinuous, or "disjunct" as described by the original authors (Engel et al., 2012; Table 2). This is also consistent with localized ground-water discharge, and not with laterally continuous deposition by a lake.

Shorelines are also conspicuously absent in the basins at Mundafan, al Hawa, and Nafud, although these locations are counted as paleolakes in compilations and in cases even incorporated into the lake status database as either high, intermediate, or low lake level stands. The one possible exception is along the east and northeast edges of al-Hawa, Yemen (Table 2), where a vague and discontinuous potential shoreline of unknown age may be present.

Roberts and Wright (1993, p. 208) presented a lake level curve for Mundafan, citing McClure (1984) as the source of the data. However, McClure himself was more conservative in his interpretation and actually did not provide such a curve (Table 2). The various levels reported by McClure (1984) could have been caused by shifting dunes and respective changes relative to the current lowest points in inter-dune basin (e.g., McClure, 1984, p. 82). In addition, most of the deposits reported from Mundafan by Rosenberg et al. (2011, and the photographs in Rosenberg et al., 2011, GSA Data Repository item 2011333 and Crassard et al., 2013; Table 2) present the characteristics of wetland discharge deposits (Table 1 ) with clear lateral horizontal transition into same-level basin margin deposits, not to a shoreline. There is no noticeable topographic change from the Mundafan marsh deposits to the organic mats at these margins. i.e., the transition is without any shoreline deposit or landform, does not allow to any depth of water, indicating shallow wetland marshy environments (Table 2).

\subsection{Marsh deposits}

All the reported deposits, which the authors may have termed "lakes", were actually viewed by the original authors more as shallow wetland environments or as deposits of occasional floods. Schulz and Whitney (1986a), for example, describe the an-Nafud beds, in an area feeding only by direct rain and without drainage basins and currently only by winter rains, as "mostly swamps" or short-lived "shallow water bodies". These water bodies were "depending on the oscillating water table... in minor scale" (i.e., not lakes), although after these descriptions, Schulz and Whitney (1986a, p. 181) still refer to them as "Holocene lakes". They claimed that paleolake existence "does not imply a pluvial period of largely augmented rainfall in every case". We refer the readers to photographs 4-6 in Schulz and Whitney (1986a, p. 188-189) and Figures 11 and 13 in Rosenberg et al. (2013, p. 116118 ) that clearly present small inter-dune sandy wetlands. If there is any distinction between the Arabia lacustrine-like deposits and those reported elsewhere, it lies in the sandier and less clayey characteristics of the Arabian deposits as can be seen in these figures. i.e., the Arabia deposits are even less characteristic lake deposits. A set of several luminescence ages provided recently by Rosenberg et al. (2013), indicate that 


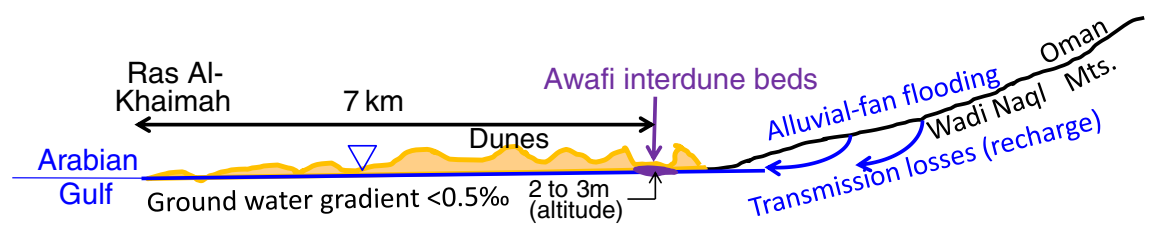

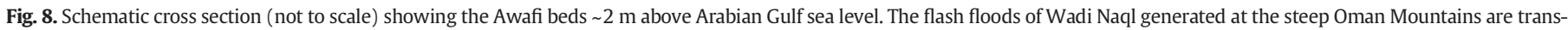

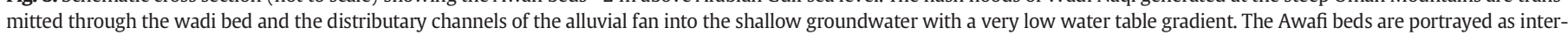

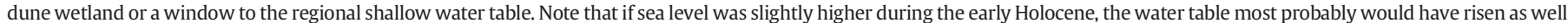

the beds documented by Schulz and Whitney (1986a) are probably related to the last interglacial and not to the early or mid-Holocene.

McClure, who extensively documented such deposits in the Rub' alKhali, claimed that the "lakes were due to temporarily perched, ephemeral and local occurrences of rainfall saturated zones" (McClure, 1984, p. 200). He advocated local wet environments perched on concentrated fines (as in pans) in inter-dunes and lowlands rather than a connection to a regional water table (as probably should be the case if all the deposits he observed were related to perennial lakes and at similar altitudes). Although McClure (1984) has termed Mundafan (the primary Rub' al-Khali lake beds) generically as "lake", in his detailed discussion, he actually pointed to shallow, partially ephemeral, wet lowlands mostly in inter-dune areas, and termed them as pans. Later, he specifically termed most of these as deposited in "ephemeral puddles" that existed only "a few months to a few years" ( see an interview with H. McClure at http://www.saudiaramcoworld.com/issue/198903/lakes.of.the.rub.alkhali.htm).

Photographs of the Mundafan (see Rosenberg et al., 2011 and ftp://rock.geosociety.org/pub/reposit/2011/2011333.pdf) and al-Hawa

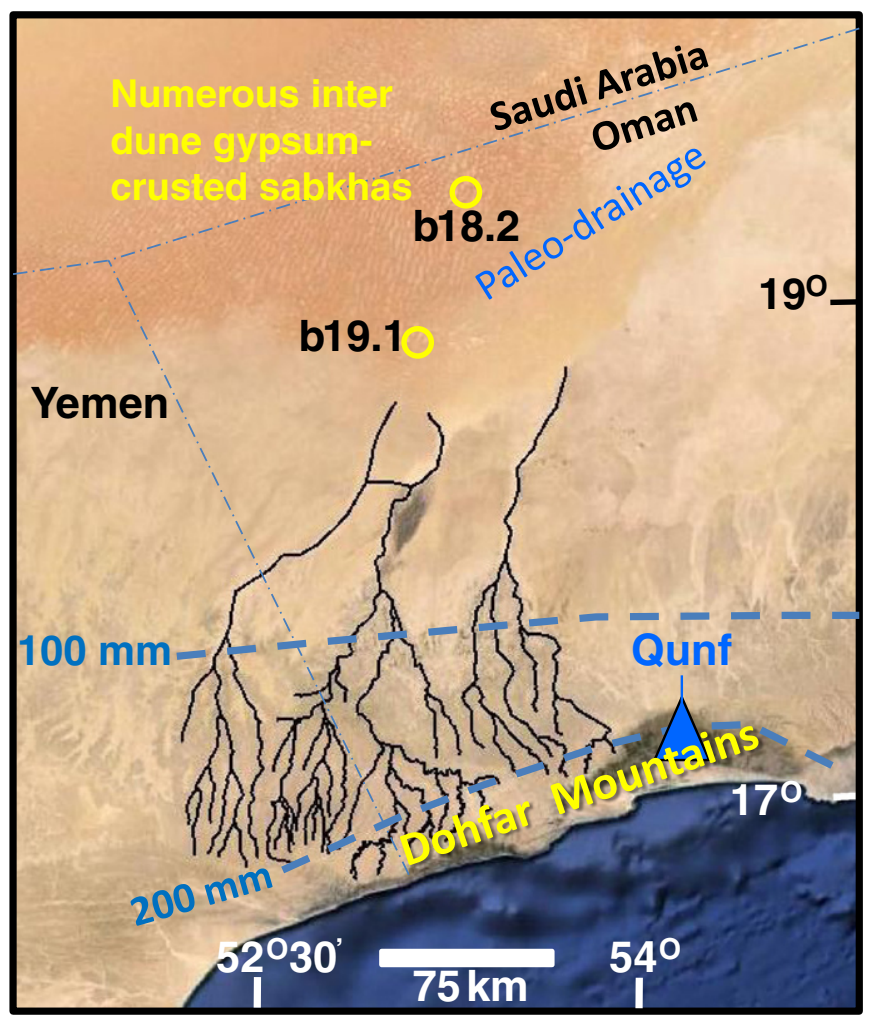

Fig. 9. Southern Oman watersheds draining the wetter elevated ( $>1000 \mathrm{~m}$ ) edges of the Arabian peninsula inland towards the Rub' al-Khali sabkhas area. Increased rainfall in the Dhofar Mountains could have been one of the causes of the numerous gypsum-crusted sabkhas (capillary zone deposits, Matter et al., in press) at the southern and eastern edges of the erg (see also Sultan et al., 2008). Note that rainfall in this mountainous edge possibly originates from the Indian Ocean. However, this is also the easternmost area in southwestern Arabia receiving moisture by the African monsoon.
(Lézine et al., 2007, p. 237) present deposits similar to wetland/marsh deposits presented by Quade and coauthors (Quade, 1986; Quade and Pratt, 1989; Quade et al., 1995, 1998, 2003, 2008; Rech et al., 2002, 2003; Pigati et al., 2009). Even if McClure (1984) argued for isolated "ephemeral puddles", Mundafan, the most significant of his reported "lake" beds, should be viewed as deposits at the edge of the Rub' al-Khali, hydrologically fed by a large drainage basin (Fig. 7) heading at somewhat wetter environment in the Red Sea margins that could have been even slightly wetter in the past and probably also delivering groundwater.

\subsection{Asymmetry in deposition}

Visible asymmetry in the distribution of clastic sediments and evaporites also assists in distinguishing lake from ground-water discharge deposits; it is valuable when associated with other indicators and is not a stand-alone argument. True lake shorelines and associated beach deposits are commonly distributed evenly around a basin at a constant elevation, whereas ground-water discharge deposits are often asymmetrically distributed, and concentrated on the side of the basin where discharge is concentrated. As an example, the asymmetric pattern of the fine-grained deposits in the Tayma basin is typical of ground-water, not lacustrine deposition (Table 1; e.g., Quade et al., 1995). The light-colored fine-grained deposits at Tayma are concentrated in an area just below the modern artesian springs, including the modern palm grove (Engel et al., 2012) that probably testifies of near surface groundwater. This suggests a paleodischarge across a confined seep-face, down slope of the springs. The presence of these deposits on the western, but not the eastern parts of the Tayma basin points to recharge from the west.

\subsection{Faunal and floral remains}

The faunal and floral remains of the basins themselves do not necessarily indicate a lake setting, but rather in most cases point to a marshier wetland environment (Table 1). These include numerous shallowwater gastropods, reed vegetation (such as Typha and Phragmites) and root casts, diverse diatoms and ostracodes that indicate occasional, but mostly shallow bodies of water (Table 2 ), and many calcareous deposits that characterize also other wetland discharge deposits. The common documentation of root casts even at basin centers indicates very shallow environments.

\subsection{Lake-budget calculations}

Paleo-rainfall can be estimated using simple hydrologic mass balance modeling, assuming paleolake areas and depths are accurately known. At steady state, hydrologic models assume that losses due to evaporation from a lake equal the gains for the lake from rainfall onto the lake plus runoff from the surrounding catchment. Such calculations are available in Arabia mainly for Tayma, and implicitly underlie interpretations from other lake basins in that region without making any calculation.

Wellbrock and Grottker (2010) and Wellbrock et al. (2011) calculated the necessary conditions to produce a Holocene lake in the Tayma basin. These include: (a) Tripling modern annual rainfall of $\leq 50 \mathrm{~mm} \mathrm{yr}^{-1}$ to 
$\sim 150 \mathrm{~mm} \mathrm{yr}^{-1}$, although even this large rainfall increase is much too low or "not enough for the development of perennial lakes in other parts of the Nafud" (Rosenberg et al., 2013, p. 118). (b) An annual runoff coefficient of $30 \%$ from the entire drainage basin, although such annual runoff coefficient is by far higher than observed in any modern data from arid lands (e.g., Supplement Table 1). (c) Drastic evaporation reduction of $>32 \%$ to $\sim 1500 \mathrm{~mm} \mathrm{yr}^{-1}$, a level of reduction that has been proposed in other lake budget calculations, but only for the much colder Pleistocene glacial times relative to present-day evaporation (e.g., Benson, 1986). In fact, Holocene temperatures in the subtropical deserts were probably slightly higher temperatures; it is also possible that it was windier as increased monsoon causes increased subsidence over north-central Arabia and air flow (see Figure 2 in Ziv et al., 2004). Perhaps the hints of dune activity and preservation in the northeast Rub' al-Khali during 7-3 ka (e.g., Atkinson et al., 2011a, p. 188, 2011b p. 104) indicate increased wind persistency; however these ages postdate the Tayma basin deposits. Higher temperature and increased advection by wind would combine to increase evaporation over terminal basins over the current estimate of $>2200 \mathrm{~mm} \mathrm{yr}^{-1}$, rather than to reduce it. All these considerations indicate that it is extremely difficult under reasonable or even under more favorable assumptions to generate a perennial deep lake in Tayma.

\subsection{Summarizing lacustrine-like indicators}

In summary, re-reading of published observations points to the lack of evidence of even intermediate lake stands in Arabia in the middle Holocene. The evidence supports mostly the existence of springs, wetmeadows, coastal sabkhas, and some marshes. This would still require an increase in rainfall in these arid to hyperarid Arabia environments, but much less than lakes will demand. For example, the minimum annual inflow needed to simply cover the surface with water would be equivalent to a depth of $2.2 \mathrm{~m}$ (the mean annual evaporation rate in Arabia) over the entire lake area. Therefore, any increase of water depth to produce a lake will demand much more incoming water. Runoff coefficients in arid to hyperarid basins are $~ 5-10 \%$ in very small drainage basins $\left(<5 \mathrm{~km}^{2}\right)$ during individual extreme storms, and even lower in larger drainage areas (Supplement Table 1). The values of annual runoff coefficients are therefore, even lower than $10 \%$. This suggests a crucial role for frequency changes of extreme storms in constructing and maintaining paleolakes in arid environments (e.g., Enzel, 1992; Enzel and Wells, 1997). Therefore, in the Arabian arid environments, maintaining water at the surface by rainfall-runoff is difficult and demands much higher amounts of rainfall than seen today. Maintaining wetlands by groundwater discharge, demands less rainfall. From first-order calculations of the minimal changes in hydrological-budget needed to form a lake, we estimated that to form an even centimeter- or decimeter-scale-deep perennial lake, the mean annual rainfall over the entire contributing drainage basins of alHawa, Mundafan, Awafi, and Tayma should be approximately 1.5, 3, 3, and 5 times than today, respectively. This is a minimum quantity and even doubling common runoff coefficients in deserts will not produce a runoff-based lake in most of the basins. In our opinion the hydrologic parameter values used by Wellbrock et al. (2011) for raising a lake in Tayma are therefore, too low (annual rainfall and evaporation) or too high (annual runoff coefficient) or, more probably, a lake did not form there. In the smaller numerous basins of an-Nafud and Rub al-Khali, with lacustrine-like deposits, these values should be even larger if perennial, several-meter deep lakes ever existed there. This indicates that to form true lakes Arabia is probably very difficult even with the proposed climatic changes.

\section{Limited vegetation changes in Arabia}

Available Holocene pollen records from Arabia are limited in number and quality (Lézine et al., 2010; Table 2). We review the data to find out if the recorded Holocene vegetation cover change supports large hydroclimatic changes in the region.

The an-Nafud pollen is similar to the present-day with some longdistance transported pollen (Schulz and Whitney, 1986a,b). However, Rosenberg et al. (2013) have shown recently that this record dates back to the last interglacial. The Mundafan pollen record has indications of grasses and mainly marsh habitat (El-Moslimany, 1983; McClure, 1984). Based on the Mundafan and the an-Nafud records, ElMoslimany (1983) argues for decreasing precipitation between Mundafan and the north Rub' al-Khali in the Middle Holocene, but the older ages recently attributed to the an-Nafud sediments and pollen (Rosenberg et al., 2013) may alter this view. The al-Hawa record is dominated by desert vegetation without a noticeable change from modern environment (Lézine et al., 1998, 2007, 2010).

The Awafi pollen record, on the other hand, seems to be the only location with a proposal for a drastic environmental change. Its record shows an expansion of savannah grassland with woodland elements, which indicate a contribution of the Indian monsoon summer rains in an area currently dominated by winter rains (Parker et al., 2004; Parker and Goudie, 2008). Lézine et al. (2010) claim however, that the proposed changes to summer rains are unsupported by the pollen data, and raised the need to reconsider the advocated slight change in rainfall amounts and argued for no change in seasonality of Holocene rainfall in that basin.

According to Lézine et al. (2010), only littoral regions in southeastern Arabia experienced noticeable vegetation changes. The pollen record from the Dead Sea, which may be considered as at Arabia's northern edge, shows a slight increase in Mediterranean winter rains in the middle Holocene (Litt et al., 2012).

To summarize, the available pollen records indicate that, at most, only limited vegetation changes occurred between the early Holocene and the present in entire Arabia (e.g., a map presented by Prentice et al., 2000; Lézine et al., 1998, 2007, 2010). This agrees with the inferences presented above that the lacustrine-like deposits in Arabia indicate only moderate Holocene hydroclimatic changes. A similar view is presented by Lézine et al. (1998, p. 297, 2007) and Lézine (2009) who noted the dichotomy between very little change indicated by the pollen record and the commonly applied concept of wetter, lake-covered Arabia. They stress that at $15^{\circ}-16^{\circ} \mathrm{N}$ in Arabia, Lézine et al. (1998, p. 297 , 2007) and Lézine (2009) did not identify the pollen that resembles the pollen indicating more humid early Holocene in North Africa, where they worked extensively, and concluded that the "increased rainfall and related runoff [from headwaters in southwest Arabia mountains] did not have any significant impact on the local [al-Hawa region] vegetation, except reed swamp [as in wetlands at lowland ends of blocked streams]". Lézine et al. $(2007,2010)$ identify the topographic barriers along the eastern Red Sea margins to the west and along the Gulf of Aden to the south as key factors in preventing a significant increase in Holocene rains from penetrating Arabia's interior.

\section{Rainfall shifts based on Speleothems}

A number of paleoclimate speleothem studies using U-Th geochronology and analysis of isotopic composition from carbonates and fluid inclusions point that the paleohydrologic changes from Arabia were cyclic with interglacial presenting wetter conditions (Burns et al., 1998, 2001; Fleitmann et al., 2003a,b, 2004, 2007, 2011; Frumkin et al., 2008; Neff et al., 2001; Shakun et al., 2007).

These changing conditions in Arabia are also supported by groundwater and rainfall geochemical analyses. For example, based on clusters of radiocarbon content in groundwater at 10,36-60, and 100 percent modern carbon (pMC), Weyhenmeyer et al. (2002) proposed three recharge episodes at 24-15 ka, 6.3-0.9 ka, and modern. Considering the complications associated with a simple transfer from concentrations to radiocarbon ages of groundwater, we still point to the lack of speleothem formation during the 24-15 ka recharge period from the Indian Ocean (compare for example Fleitmann et al., 2007; Weyhenmeyer et al., 
2002). We also reiterate the question raised by Weyhenmeyer et al. (2000) to the absence of $10-36$ pMC from the aquifer samples (Weyhenmeyer et al., 2002); such values should have existed if the early Holocene was the wettest episode. They explain this gap in their results by the little room available in the coastal aquifer for the recharging water during the early Holocene sea level rise (Weyhenmeyer et al., 2000 p. 844).

Holocene-age speleothems are mainly restricted to the elevated areas in the southern and southeastern edges of the peninsula (Fig. 6) (e.g., Fleitmann et al., 2007) and a few from Socotra Island (Shakun et al., 2007; Van Rampelbergh et al., 2013); none was recovered from inland peninsula locations, away from coastal ranges (Fleitmann et al., 2004; Frumkin et al., 2008).

The rainfall amounts necessary for speleothem formation on the southern fringes of the Arabian Peninsula are basically unknown (and probably depend on individual cave recharging conditions), but the underlying assumption is that it was greater than today, although few caves do drip even today (Burns et al., 2001; Fleitmann et al., 2003a,b, 2011). In the Negev, Vaks et al. (2010) assumed that a mean annual (in this case it is actually winter) precipitation of $300-350 \mathrm{~mm}$ is necessary for speleothem formation. Fleitmann et al. (2011) suggest a doubling or more of the current rainfall in Hoti and Mukalla Caves to produce the MIS 1 and MIS 5 speleothems in southern Arabia. These rainfall increases are attributed to increased moisture transport into Arabia from the northern Indian Ocean through the southwest Indian monsoon (Fleitmann et al., 2003b; Weyhenmeyer et al., 2002).

The longest Holocene records in Socotra Island are from its eastern (Hoq Cave, since 7 ka; Van Rampelbergh et al., 2013) and western (Dimarshim Cave; since 4.5 ka; Fleitmann et al., 2007; Shakun et al., 2007) parts of the island that currently are receiving winter rains and late spring (April-May) rains, respectively. Socotra Island does not receive rain during the heart of the southwest Indian monsoon months (July-August; Scholte and De Geest, 2010). The winter rains are delivered to northeast Socotra by the northeast winter monsoon winds with amounts increasing with altitude (Scholte and De Geest, 2010; Van Rampelbergh et al., 2013); this may indicate the potential of the northeast winter monsoon to generate rainfall where it flows over large stretches of water (as in Gulf of Oman upwind of the Oman Mountains).

The peninsular Arabian caves, such as Hoti, Qunf, and Mukalla, that contain early-middle Holocene speleothems (Burns et al., 1998; Fleitmann et al., 2011) are located in mountainous areas at altitudes between 650 and $1500 \mathrm{~m}$. The annual precipitation for the two caves according to the CRU TS 2.1 gridded dataset (Mitchell and Jones, 2005) are $>120$ and $\sim 170 \mathrm{~mm} \mathrm{yr}^{-1}$, respectively. However, current mean annual rainfall in these regions strongly increases with altitude (e.g., Al-Rawas and Valeo, 2009; Kwarteng et al., 2009; Weyhenmeyer et al., 2002), and the higher altitudes of the recharge areas of Hoti and Mukalla Caves may thus receive even today $250-350$ and $>300 \mathrm{~mm} \mathrm{yr}^{-1}$, respectively. The exact amounts are unknown and the actual increase should be more carefully estimated.

Isotopic composition of the fluid inclusions in the Hoti Cave speleothems indicates Indian Ocean origin for the speleothem-forming water, although at present this region gets most of its rainfall in the boreal winter and spring (Fig. 2). Rains from the south or with southern isotopic composition (in contrast to a Mediterranean moisture sources) were proposed to be generated by three potential ways: from the ISM, from regional and/or local convective orographic rain cells, or by northward-moving Arabian Sea tropical cyclones (Fleitmann et al., 2003b; Weyhenmeyer et al., 2002), which have similar isotopic composition to storms over India. Of these mechanisms, Fleitmann et al. (2003a,b) favored increased ISM rains and associated shifts in ITCZ. Their main argument for this preference was the coeval existence of the abovementioned lakes in Arabia (Fleitmann et al., 2003b, p. 231) challenged here. An additional potential water source, not considered in the existing literature, is the nearby Gulf of Oman (primarily for
Hoti Cave), which should have an isotopic composition similar to the Indian Ocean, from which the gulf is extended. This source should be examined and considered as effective under the winter northeast monsoon and may explain episodes of significant recharge in coastal northern Oman.

In summary, Holocene speleothems are confined to the coastal mountain fringes of the southern Arabian Peninsula (i.e. within $\sim 100 \mathrm{~km}$ from the coast) and do show somewhat wetter conditions, in the middle Holocene. The water source could be increased ISM rains (Fleitmann et al., 2003a,b) or Arabian Sea cyclones (Weyhenmeyer et al., 2002), or potentially even winter air flow over the Gulf of Oman.

In contrast with these near-coast Holocene speleothems, only speleothems predating $300 \mathrm{ka}$ are found (Fleitmann et al., 2004) in a series of caves in north-central Arabia (Kh, SC, BL, and SG in Fig. 6); younger speleothems are conspicuously absent. Frumkin et al. (2008) used the age distribution of these and other speleothems from the Levant to Arabia to demonstrate the distinct two zones of speleothem formation and their relationships to moisture sources: Mediterranean moisture producing the Levant-northernmost Arabia speleothems and southern sub-tropical moisture producing the extreme southern Arabian Peninsula speleothems. The restricted spatial and temporal distribution of speleothems indicate that there is no overlap between these two sources for at least 300,000 years; i.e. for at least three glacial-interglacial cycles. This points to very scanty rainfall in central and northern Arabia and should be in the focus of all proposals for monsoon rainfall in Arabia during earlier interglacial.

\section{Middle Holocene regional hydroclimatology and its implications}

\subsection{Hydroclimatic pattern}

Our re-examination of the hydroclimatic proxy evidence suggests that during the middle Holocene, the southwest and southeast Arabian Peninsula were somewhat wetter than today but the core of the peninsula remained dry, as in the previous 300,000 years. Increased rainfall in the southwest, was most likely related to intensification in the African summer monsoon rain incursion into Arabia. Northwestern Arabia could have been impacted also by more frequent winter and spring tropical plumes that affect the area today (Fig. 6; and e.g., Barth and Steinkohl, 2004; Tubi and Dayan, 2014). It seems that only south-easternmost Arabia could have been impacted by small shifts in the Somali Jet that brought summer rains to that region.

An increase in rainfall amounts on the Yemen highlands, as a result of the more active African monsoon, would imply increased rainfall amounts farther east (i.e. shifting isohyets to the east), covering larger portions of headwaters of the drainage basins feeding Mundafan and al-Hawa (Figs. 7 and 8). This feeding was either direct by floods or indirectly by increased recharge to the aquifers through transmission losses. Our view is that rainfall-related environmental conditions in the interior of Arabia or even just north of the southern coastal ranges were not much different than today during the midHolocene (and to that extent also in earlier interglacials). Based on the evidence presented in this study, atmospheric circulation patterns and rainfall increase associated with such changes were apparently much more moderate than proposed until now. To associate atmospheric causes of hydrologic changes in northern Arabia with those of southern Arabia is probably against the climate dynamics that controls the region's hydrological cycle.

Current rainfall in north and east Saudi Arabia and Oman is highly influenced by winter rainfall in the form of Mediterranean cyclones and associated formation of new cyclonic depressions in front of the Zagros Mountains or winter-spring tropical plumes (Barth and Steinkohl, 2004; Rubin et al., 2007; Tubi and Dayan, 2014). If the seasonality and location of current rainfall patterns have anything to teach us, increased 
winter rainfall must remain as a plausible explanation for the minor paleohydrologic change observed there. In the future, pollen from plants sensitive to summer vs. winter-early spring rains should be carefully examined in this regard.

\subsection{Constraints on early to mid-Holocene climate}

The mechanisms that control monsoon precipitation and the latitudinal and longitudinal extent of the convection over land are complex and involve radiative heating of the land surface, moisture transport from the ocean, feedback with the land surface (related to changes in ground moisture content and vegetation cover), and circulation response to the convection itself (e.g., Chou and Neelin, 2003). The impacts of orography (East Africa, Yemen and Southern Arabia highlands, and Oman Mountains) and that of remotely forced changes in atmospheric stability and large-scale subsidence are also important (Rodwell and Hoskins, 1996). All these diverse controls of precipitation should be considered when evaluating the response to changes in insolation of the Indian and African monsoons and their extension into the Arabian Peninsula. Untangling their relative significance is a task for climate models (e.g., Braconnot et al., 2007a, b, 2008; Su and Neelin, 2005; Texier et al., 2000). Such model simulations are typically forced with insolation conditions of the midHolocene ( $6 \mathrm{ka}$ ) to purposely avoid the early part (11-9 ka) when the Northern Hemisphere climate was still influenced by relatively large ice sheets. These model runs are generally compared to the same model simulations forced with pre-industrial conditions, which are also used to verify the models' fidelity. Surprisingly, these pre-industrial model runs erroneously indicate summer rainfall in peninsula's areas that do not experience such rainfall season at all; this may indicate a bias in the results toward a larger increase in summer rainfall. In some cases, various mechanisms, such as surface vegetation cover and sea surface temperatures are modified to test their relative impact on precipitation (Braconnot et al., 2007a,b).

The experiments conducted so far support the notion that some expansion of the rainfall into the southern interior could have occurred in the mid-Holocene (Fig. 2 in Braconnot et al., 2007b), associated with the deeper penetration of the East African summer monsoon into the Arabian Peninsula (Fig. 10). The latter is supported by the more recent experiments of Herold and Lohmann (2009) who integrated a model with the last interglacial (Eemian interglacial, $125 \mathrm{ka}$ ) insolation conditions (see Figs. 2 and 3 in Herold and Lohmann, 2009). When applying the latter to the mid-Holocene case and recognizing the present-day high correlation between East Africa and southwest Arabia rains (Fig. 8), one has to account for the significantly lower level of Northern Hemisphere summer insolation during that time (about half the Eemian level, see Braconnot et al., 2008). Nevertheless, Herold and Lohmann show that the increased Arabian Peninsula precipitation during the Eemian is associated with a stronger eastward transport of moisture from the tropical Atlantic in the core of the African summer monsoon (an assumption

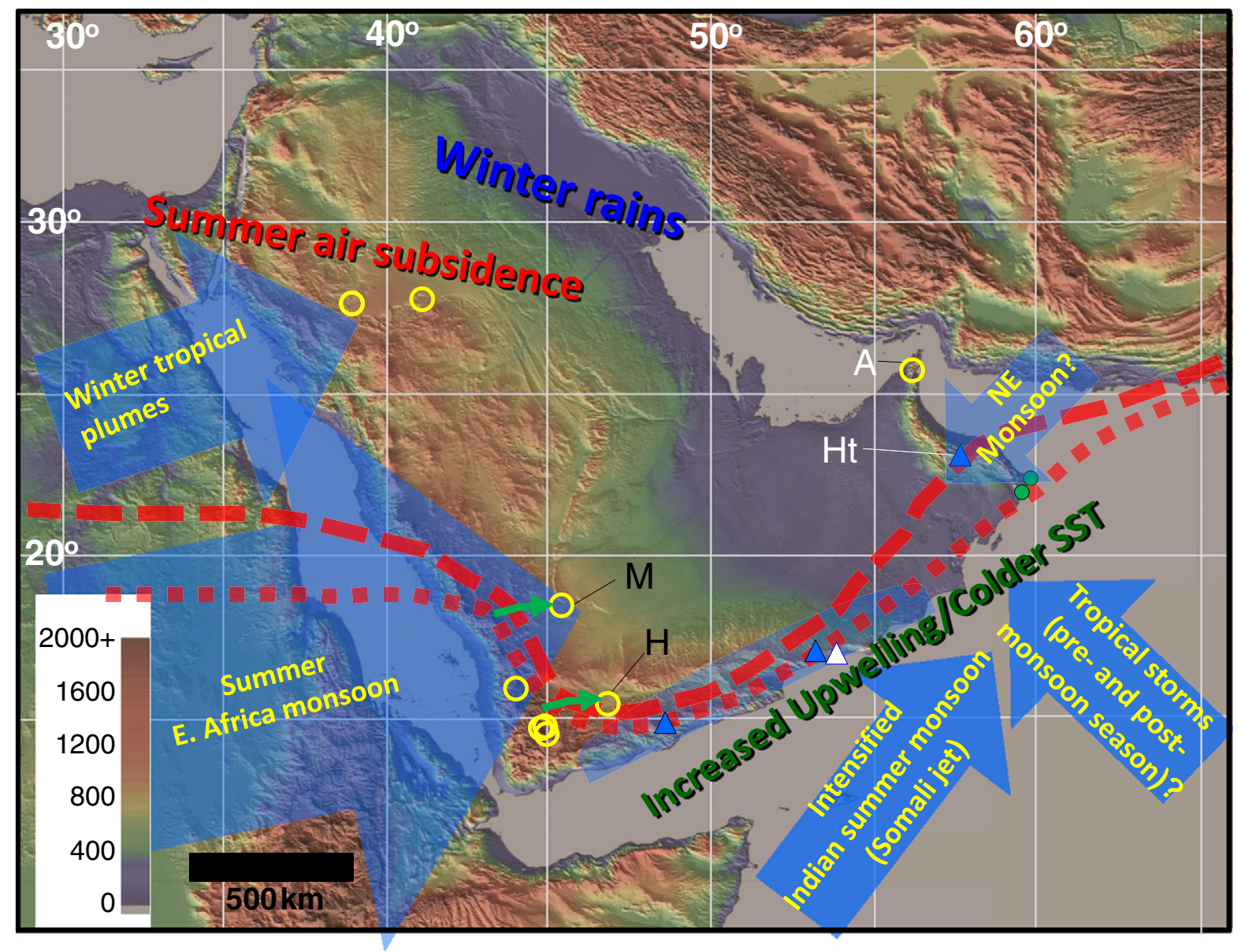

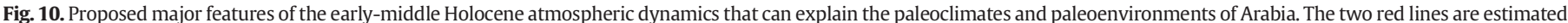

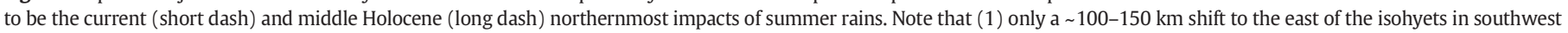

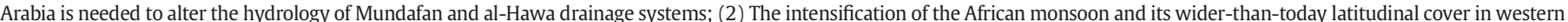

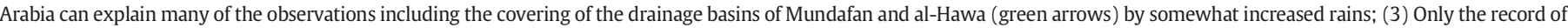

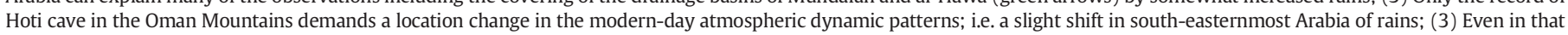
region the impact of direct moisture contribution from the Gulf of Oman should remain as a viable alternative for future research. 
consistent with the strong zonal coherence of the African summer monsoon, see Fig. 4). The mid-Holocene experiments cited above also show that over the southern peninsula rainfall amounts increase by $40-$ $240 \mathrm{~mm}$ during the entire summer season. The largest increase occurs over the mountains in southwest Arabia, where annual rainfall amounts are relatively high today reaching $>500 \mathrm{~mm}$ (Fig. 1); there the models project an increase to nearly $800 \mathrm{~mm}$ per summer season. Further east, along and across the coastal mountains the increase projected by the models is by far smaller, between $\sim 100$ and $160 \mathrm{~mm}$ for the entire summer season. The degree of spread inland is less certain because the models' coarse resolution, which affects both the presentation of the topography and the latitudinal extent of the rainfall. Herold and Lohmann show that insolation-induced summer rainfall would have increased over the southwestern peninsula and evaporation rates decrease due to the related increase in cloudiness. Thus overall precipitation-evaporation difference increases and more water is available for surface runoff and storage, but still, evaporation remains high.

The modeling results on the link between intensified summer precipitations in the Sahel during high insolation are backed by observations showing that lakes around the Ethiopian Highlands stood at higher level during the early- to middle Holocene (Gasse and Roberts, 2005); unlike in Arabia, these lakes, however, present clear Holocene shorelines.

\subsection{Southwest monsoon winds and upwelling off-Oman assist keeping Arabia dry}

As indicated above (Section 2) the low-level Somali jet is influenced by the elevated topography of the Ethiopian Highlands (Fig. 1A). At Quaternary time scales this "deflection" by the blocking topography of the Ethiopian Highlands permanently prevents this jet from curving farther north over Africa to enter Arabia beyond its southern edges. Therefore, the Somali jet flow direction and its moisture transport during the mid-Holocene (and earlier Quaternary times) could be viewed as at similar location as today's (e.g., Fig. 1A), although it could have occurred with increased wind speed (e.g., Bassinot et al., 2011).

Comparatively little of the moisture transport related to the Somali jet converges over the coasts of Africa (e.g., arid Somali experiences practically no summer rain; Fig. 2) and the Arabian Peninsula. The Arabian Sea itself is a region of moisture transport divergence forming a minimum rainfall zone between Africa in the west and the Indian subcontinent to the east. The Indian Ocean marine ITCZ lies far to the south, at or close to the equator (Figs. 1 and 3). Notably, the Indian summer monsoon (i.e., the Somali jet) winds also force upwelling and sea surface cooling along the southern Arabia coast. This cooling further stabilizes the coastal atmosphere (e.g., Evan and Camargo, 2011) that limits moisture transport inland to Oman.

\subsubsection{Upwelling off-southern Oman}

As the Somali jet flows sub-parallel to the coast of southern Arabia (e.g., Turner and Annamalai, 2012), its related upwelling off that coast cools sea surface temperatures (SSTs). The main proxies of oceanic upwelling at the continental margin of southern Oman, and therefore direct indicators of surface winds associated with the Somali jet in the Arabian Sea are variations in the abundance of the planktonic foraminifer Globigerina bulloides. The percentage abundances of G. bulloides are highly and positively correlated with rates of coastal upwelling, nutrient availability, and cooler SSTs (e.g., Overpeck et al., 1996; Peterson et al., 1991; Prell, 1984; Prell et al., 1990; Sirocko et al., 1993). This upwelling index retrieved in cores recovered off the southern Arabia coast, indicates increased mid-Holocene upwelling and cooler SSTs between 9.5 and 5.5 ka (e.g., extensive discussion in Overpeck et al., 1996; Prell and Van Campo, 1986). The intensity of upwelling (and colder SSTs) is tied, via wind-stress-forced Ekman flow, to the strength of the southwest monsoon winds - the surface expression of the Somali jet (Anderson and Prell, 1992, 1993; Bassinot et al., 2011; Prell et al.,
1990). Therefore, the intensification of the early and middle Holocene monsoon winds is probably the result of the more intense and/or persistent Somali jet component of the ISM. As in other hyperarid coastal deserts forced by Ekman-flow and strong upwelling (e.g., Namib, Atacama, NE Sahara and in our case also Somali in coastal Horn of Africa), the resulted colder SSTs and intensify ISM winds are expected to exert a negative influence on rainfall along the southern coast of the Arabian Peninsula. By contrast, in peninsular India and especially at its western edge, the rains will increase with stronger winds of the Somali jet (Halpern and Woiceshyn, 2001). This may be part of the explanation for some of the regional climatic contrasts in the areas affected by the ISM (e.g., Staubwasser, 2006).

The amplified upwelling along the southern Arabia coast could have also reduced the frequency and impact of northern Arabian Sea tropical cyclones, as it currently does during the heart (July-Sept) of the summer monsoon season (Evan and Camargo, 2011). These Arabian Sea cyclones were proposed as a potential source of rainfall for late Pleistocene recharge of coastal eastern Oman aquifers (Weyhenmeyer et al., 2002). This can be a plausible scenario under a weaker summer monsoon and therefore, reduced upwelling and possibly higher SSTs off Oman; recharge contribution (i.e. precipitation) during the early and middle Holocene was much reduced in Oman (Weyhenmeyer et al., 2002), in agreement with upwelling and lowered SSTs limiting cyclone generation in the Arabian Sea under intensified Somali jet. The above atmosphere-ocean circulation indicates that the ISM reduces rather than enhances rains in most of the Arabian Peninsula whereas the African monsoon played a larger role as perhaps also the winter northeast monsoon and the winter-spring tropical plumes.

\subsection{A shift in ITCZ?}

As indicated in the Introduction, the prevailing assumption in many paleoclimatological studies is that the insolation-induced changes in summer rainfall in the Arabian Peninsula are associated with the global zonal movement of the ITCZ north and south in boreal summer and winter, respectively. It is therefore important to note that the summer rains along the peninsula's southern mountainous region are disconnected with the boreal summer Indian Ocean ITCZ. The latter appears as a westward extension of the eastern Indian Ocean ITCZ and is located far south of the peninsula and rarely north of the equator; during extreme cases the ITCZ reaches the equator or the maximum latitude of $5^{\circ} \mathrm{N}$ (Fig. 3).

The global ITCZ, the convection zone that is associated with (in fact constitutes) the ascending branch of the Hadley Circulation is interrupted over the Indian Ocean due to the dominating influence of the Indian Ocean Monsoon and the massive convection center over the western tropical Pacific (Waliser and Gautier, 1993; Waliser and Somerville, 1994). Overall, the Northern Hemisphere Hadley circulation cell of the boreal summer is very weak and its rising branch and associated ITCZ convection is located usually south of $5^{\circ} \mathrm{N}$, depending on longitude (Dima and Wallace, 2003; Tanaka et al., 2004) (Figs. 1 and 3). This rising branch preferentially feeds the southern Hadley Cell. Moreover, over the Indian Ocean sector, the divergent summer circulation (that which is associated with the vertical mass overturning) is not aligned with the global Hadley Circulation, i.e., displaying association with the zone of low-level convergence and upper-level divergence stretching roughly parallel to the equator. Rather, convergence and divergence zones are aligned almost perpendicular to the equator, in a longitudinal direction (Tanaka et al., 2004). This transverse (meridional) circulation cell, with its rising branch extending over a broad region, from the Bay of Bengal (with some ascending air also over western peninsular India) to the west tropical Pacific, and sinking branch over North Africa and the eastern Mediterranean, is part of the tropical Walker Circulation (Texier et al., 2000; Tanaka et al., 2004) and is driven by the summer monsoon and the massive Indo-Pacific convection center. The summer rains in southwestern Arabia today 
are in fact not related to the Indian Ocean ITCZ but rather, as argued above, primarily to the African summer monsoon. During maximum insolation, when both the ISM and the African summer monsoon were stronger due mainly to enhanced summer warming over land, it is hard to envision that the Indian Ocean ITCZ has intensified or moved north. Rather, it is the latitudinal expansion and/or northward movement of the African summer monsoon that has led to or has been associated with the intensification and expansion of rainfall over southern and western Arabia. Indeed, general circulation models of the midHolocene and the last interglacial confirm this scenario (more about this below in Section 7.5).

\subsection{Implications to pre-Holocene interglacial climates of Arabia}

The proposed wetter early-middle Holocene climate in Arabia is commonly extended to pre-Holocene, insolation maxima interglacial times. This practice is based on the lacustrine (or wetlands as proposed above) and speleothems (e.g. Rosenberg et al., 2011; Fleitmann et al., 2007). We do not claim to the contrary. However, we discuss below the abovementioned climatic causes in explanations of earlier interglacial climatology and the degree of hydrologic impacts and changes. The regional physiography and the main ocean and atmosphere circulations of the northern Arabian Sea and its surrounding continents are assumed similar to both the early to middle Holocene and earlier interglacials. For example, as the Somali jet is a low-level jet ( $<2 \mathrm{~km}$ in the atmosphere), it cannot penetrate through and across the elevated southwestern edges of Arabia and northeastern Africa; i.e., the Somali jet (the actual ISM wind) is practically blocked by the regional physiography and elevated topography from entering Arabia. Questioning the proposed mechanisms of changing pre-Holocene, interglacial Arabia climate is, therefore, important as they are commonly hypothesized as facilitators of human migration out of Africa and across Arabia. Our main point here is that claims to increased wetness in Arabia through ISM rains, in cases even all the way to the peninsula's northern hyperarid edges, should be founded on better understanding of climatology, field data and clearer interpretations. To tie this increased wetness, if ever occurred in the magnitude proposed, to the ISM is in many ways contradicting current understanding of the main topographic, oceanographic and atmospheric forcing. For example, based on scarce field evidence and $U-T h$ ages, an enormous Eemian (and earlier interglacials) open water lake ( $40 \mathrm{~m}$ deep, $>2000 \mathrm{~km}^{2}$ ) was proposed by Petit-Maire et al. (2010) to fill the Mudawwara depression in northern Arabia (at the Jordanian-Saudi Arabia border); its formation was associated by Petit-Maire et al. (2010) with drastic shift to the north of the ISM. More recent studies (Catlett, 2014; Catlett et al., 2013; Rech et al., $2014,2016)$ challenged the existence of such a lake during the last interglacial, claiming that (a) the U-Th ages on these deposits are problematic and therefore, Mudawwara deposits "should not be used as evidence for enhanced precipitation [and lake]... during Pleistocene interglacial periods" (Catlett et al., 2013), and (b) based on sedimentology of the deposits in Mudawwara, their spatial distribution, lateral facies assemblages, the presence of archeological materials, root voids, and other trace fossils, as well as invertebrate fossil assemblages, Mudawwara (as well as other basins in southern Jordan) were actually fed by ground-water discharge and were not lakes (Rech et al., 2014).

Even the recent revision of the chronology of Mundafan and anNafud beds (Rosenberg et al., 2011, 2013), indicating that they are not Holocene deposits and probably related to the somewhat wetter last interglacial in western Arabia, does not provide evidence of increased Indian summer monsoon. More so, Rosenberg et al. (2013) apply the modeling results of Herold and Lohmann (2009) to indicate a growing importance of the African monsoon in western Arabia. Additional modeling by Kutzbach et al. (2014, see their Fig. 4a) shows an extension of the African monsoon summer rains into Arabia during last interglacial maximum insolation ( $125 \mathrm{ka})$ in a spatial pattern that mimics presentday correlation of rainfall across Africa and Arabia (Fig. 8).
When analyzing interglacial climatology (of low to mid-latitudes and mainly the early and middle Holocene and the last interglacial) most modeling and analyses focus primarily on the summer circulation and practically avoid the impacts of winter rainfall during interglacials as potential causes of the observed hydroclimatic changes. A recent exception (Kutzbach et al., 2014) to this dominated summercirculation-interglacial climates approach has implication to Arabia paleoclimatology. They focus on atmospheric circulation over the Mediterranean and Middle East during maximum Northern Hemisphere seasonality ( $\sim 125 \mathrm{ka}$ ) and comparing it with the later ( $110 \mathrm{ka}$ ) minimum seasonality. They point to the importance of winter circulation in addition to summer circulation. Among the features observed in their results (but not discussed there) is a noticeable increase of airflow into northwestern Arabia and the Levant from the eastern tropical $\left(20^{\circ}-30^{\circ} \mathrm{N}\right)$ Atlantic, sub-paralleling the equator (Fig. 3 in Kutzbach et al., 2014); this airflow trajectory is observed during modern precipitating winter-spring tropical plumes impacting northwestern Arabia (e.g., Rubin et al., 2007; Tubi and Dayan, 2014). i.e., in addition to the African summer monsoon expanding across the Red Sea into Arabia, heavy rainfall can reach northwestern Arabia (and in this case even northeastern Egypt, Sinai, Negev, and southern Jordan) during the winter season; both the African summer monsoon and the winter-spring tropical plumes are plausible alternatives to the ISM as primary factors in increasing regional rainfall. The two season rainfall may have been another alternative to the somewhat wetter Arabia during the last interglacial relative to the early and middle Holocene.

\subsection{Implication for human-lacustrine association in Arabia}

Recently, new research connects prehistoric (late Pleistocene and Holocene) human crossing and habitation of Arabia with potentially improved hydroclimatic conditions. These findings are exciting, but we stress that there is no need for lakes to explain the distribution of humans in Arabia; wetlands are sufficient or even superior to lakes in providing needed resources to humans and animals (e.g., Ashley et al., 2010a,b). This distinction is important as the mixed terminology used: lake, lacustrine deposit or environment, dry playa lake or ephemeral lake should be supported by evidence that is then associated with magnitudes of hydroclimatic changes. For example, the recent identifications of human artifacts and faunal remains around and in flat lowland basins in Arabia were associated with the existence of lakes during various interglacials (e.g. Petraglia et al., 2011; Crassard et al., 2013); these terms (lakes) should not be lightly used as they are extremely important in regional paleoclimatology. Efforts in studying paleoenvironments in Arabia (and elsewhere in the world current deserts) should go beyond the identification of lacustrine-like deposits, which consist of many types and deposited in diverse environments, and, if possible, present evidence for a water depth of at least a few meters; such deeper perennial or short-lived water bodies are crucial for scaling paleoclimatic interpretations.

\section{Conclusions}

1) The sedimentologic, floral and faunal characteristics of lacustrinelike deposits in Arabia point to shallow wetland paleoenvironments, not lakes. At steady state, smaller surface areas of wetlands versus lakes require a much smaller increase in Arabian rainfall than the 3- or 4-fold increase envisioned by other studies. Since lakes did not occupy these basins, lake budget calculations are not a suitable approach to estimating changes in rainfall-runoff-evaporation in Arabia. Although these wetter environments indicate a wetter climate, the hydroclimatic changes needed to explain them are much smaller than those required for lakes. Furthermore, the lake basins of Arabia lack evidence of shorelines, the main criteria for even a shallow lake. 
2) The creation of the observed depositional environments does not require (a) substantial reduction in annual evaporation or (b) an order-of-magnitude increase in annual runoff coefficients. From this we conclude that modest, not dramatic, increase in rainfall could have accounted for the early- to mid-Holocene paleowetland development in Arabia.

3) The spatial distribution of the rare Holocene speleothems and pollen spectra recovered from Arabia support the conclusion from the lacustrine-like deposits of only modestly wetter early-middle Holocene climates. Over most of Arabia, which currently experiences low rainfall amounts and winter rain maxima, major paleohydrologic changes are not apparent. The most notable changes are at the southern edges of the peninsula.

4) The Indian Ocean has had little impact on Arabia beyond the southern edges of the peninsula. Both the Indian summer monsoon and the ITCZ are not the main atmospheric features bringing additional moisture into Arabia. The intensified summer southwest monsoon winds, manifested in the intensification of the low-level Somali jet flowing sub-parallel to Arabia southern edge, delivered substantially more moisture eastward, towards India, but far less dramatically northward into Arabia. This is primarily due to the stabilizing effect of increased upwelling (in response to the Somali jet) off-southern Oman and the associated colder coastal SSTs. That said, southern sources of moisture could have accounts for rainfall increases in the mountainous southern fringes of Arabia due to orographic lifting. For interior Arabia and mainly at its northwestern edges, increases in wintertime rainfall, as opposed to southerly summer moisture, should be seriously considered and tested using seasonality as potentially inferred from pollen. This first should be verified in eastern Mediterranean and Levant records that are located atmospherically upstream of Arabia or are affected by tropical plumes at their southern edges.

5) The most pronounced increase in precipitation occurred in southwestern Arabia. There, during earlier Holocene, the intensification of the African monsoon and not of the Indian summer monsoon, affected a wider latitudinal range east of the Red Sea and delivered more rainfall across the Red Sea highlands to impact the drainage basin flowing east into the sandy deserts of Arabia. Tropical plumes can bring rainfall to northwestern Arabia. i.e., it should be considered that neither a single season nor a single climatic pattern controls the proposed minor hydroclimate changes in Arabia.

6) Research should focus on the often-ignored disagreement between GCM simulations of early- to mid-Holocene (and earlier interglacials) climates and earlier conclusions regarding Arabian Peninsula rainfall. Specifically, the proposed extensive latitudinal shifts of the ITCZ and considerable intensification of rainfall, based on the earlier inferences of lake, pollen and speleothems records and the GCMs results of e.g., Braconnot et al. (2004, 2007a), Bosmans et al. (2012), Marzin and Braconnot (2009), Zhao et al. (2005) can be reconciled by our reevaluation of the data.

\section{Acknowledgments}

Dr. Ron Kahana (Met Office Hadley Centre, Exeter) provided an excellent critical colleague review. We thank H. Gildor, J. Pigati, and J. Rech for fruitful discussions over the years on the latitudinal changes in monsoons and ITCZ and on the meaning of "lacustrine environments". We also thank the GCP editors for inviting this review and the editor (T. Cronin) and an anonymous reviewer for their efforts in editing this long manuscript. We acknowledge financial support from LamontDoherty Earth Observatory (Columbia University) to YE and The Lady Davis Foundation and The Fredy and Nadine Herrmann Institute of Earth Sciences (The Hebrew University of Jerusalem) to YK and JQ that enabled mutual visits to focus on this work. YK was also supported by the U.S. National Oceanic and Atmospheric Administration (NOAA) grant NA100AR4320137. YE research is funded by the Israel Science
Foundation-The Dead Sea Deep Core, Center of Excellence (Grant No. 1436/14). This article carries the designated LDEO publication \# 7890.

\section{Appendix A. Supplementary data}

Supplementary data to this article can be found online at http://dx. doi.org/10.1016/j.gloplacha.2015.03.004.

\section{References}

Adams, K., Wesnousky, S., 1998. Shoreline processes and the age of the Lake Lahontan highstand in Jessup embayment, Nevada. Geol. Soc. Am. Bull. 110, 1318-1332.

Al Farraj, A., Harvey, A.M., 2000. Desert pavement characteristics on wadi terrace and alluvial fan surfaces: Wadi Al-Bih, UAE and Oman. Geomorphology 35, 279-297.

Al Farraj, A., Harvey, A.M., 2004. Late Quaternary interactions between aeolian and fluvial processes: a case study in the northern UAE. J. Arid Environ. 56, 235-248.

Almazroui, M., 2011. Calibration of TRMM rainfall climatology over Saudi Arabia during 1998-2009. Atmos. Res. 99, 400-414.

Almazroui, M., Islam, N.M., Athar, H., Jones, P.D., Rahman, M.A., 2012. Recent climate change in the Arabian Peninsula: annual rainfall and temperature analysis of Saudi Arabia for 1978-2009. Int. J. Climatol. 32, 953-966.

Almazroui, M., Abid, M.A., Athar, H., Islam, M.N., Eshan, M.A., 2013. Interannual variability of rainfall over the Arabian Peninsula using the IPCC AR4 Global Climate Models. Int. J. Climatol. 33, 2328-2340.

Alpert, P., Osetinsky, I., Ziv, B., Shafir, H., 2004. Semi-objective classification for daily synoptic systems: application to the Eastern Mediterranean climate change. Int. J. Climatol. 24 (8), 1001-1011.

Al-Rawas, G.A., Valeo, C., 2009. Characteristics of rainstorm temporal distributions in arid mountainous and coastal regions. J. Hydrol. 376, 318-326.

Anderson, D.M., Prell, W.L., 1992. The structure of the southwest monsoon winds over the Arabian Sea during the late Quaternary - observations, simulation, and marine geologic evidence. J. Geophys. Res. Oceans 97, 15481-15487.

Anderson, D.M., Prell, W.L., 1993. A 300 KYR record of upwelling off Oman during the late Quaternary - evidence of the Asian southwest monsoon. Paleoceanography 8 193-208.

Anselmatti, F.S., Ariztegui, D., de Batist, M., Gephardt, A.C., Haberzatettl, T., Niessen, F., Ohlendorf, C., Zolitschka, B., 2006. Environmental history of southern Patagonia by seismic stratigraphy of Laguna Potrok Aike. Sedimentology 56, 873-892.

Ashley, G.M., Barboni, D., Domínguez-Rodrigo, M., Bunn, H.T., Mabulla, A.Z.P., Diez-Martin, F., Barba, R., Baquedano, E., 2010a. A spring and wooded habitat at FLK Zinj and their relevance to origins of human behavior. Quat. Res. 74, 304-314.

Ashley, G.M., Barboni, D., Domínguez-Rodrigo, M., Bunn, H.T., Mabulla, A.Z.P., Diez-Martin, F., Barba, R., Baquedano, E., 2010b. Paleoenvironmental and paleoecological reconstruction of a freshwater oasis in savannah grassland at FLK North, Olduvai Gorge, Tanzania. Quat. Res. 74, 333-343.

Atkinson, O.A.C., Thomas, D.S.G., Goudie, A.S., Parker, A.G., 2011a. Holocene development of multiple dune generations in the northeast Rub'al-Khali, United Arab Emirates. The Holocene 22, 179-189.

Atkinson, O.A.C., Thomas, D.S.G., Goudie, A.S., Baily, R.M., 2011b. Late Quaternary chronology of major dune ridge development in the northeast Rub' al-Khali, United Arab Emirates. Quat. Res. 76, 93-105.

Barth, H.J., Steinkohl, F., 2004. Origin of winter precipitation in the central coastal lowlands of Saudi Arabia. J. Arid Environ. 57, 101-115.

Bartov, Y., Bookman, R., Enzel, Y., 2006. Current depositional environments at the Dead Sea margins as indicators of its past levels. In: Enzel, Y., Agnon, Stein, M. (Eds.), New Frontiers in Dead Sea Paleoenvironmental Research. Geological Society of America Special Paper 401, pp. 127-140.

Bassinot, F.C., Marzin, C., Braconnot, P., Marti, O., Mathien-Blard, E., Lombard, F., Bopp, L, 2011. Holocene evolution of summer winds and marine productivity in the tropical Indian Ocean in response to insolation forcing: data-model comparison. Clim. Past 7, 815-829.

Benson, L.V., 1986. The sensitivity of evaporation rate to climate change-results of an energy-balance approach. U.S. Geological Survey Water-Resources Investigation Rep. 86-4148. 1-22, U.S. Department of the Interior, U.S. Geological Survey, Denver Colorado (40 pp.).

Benson, L.V., Paillet, F.L., 1989. The use of total lake-surface area as an indicator of climatic change: examples from the Lahontan basin. Quat. Res. 32, 262-275.

Berger, J.F., Bravard, J.P., Purdue, L., Benoist, A., Mouton, M., Breamer, F., 2012. Rivers of the Hadarmawt watershed (Yemen) during the Holocene: clues for late functioning. Quat. Int. 266, 142-161.

Bosmans, J.H.C., Drijfhout, S.S., Tuenter, E., Lourens, L.J., Hilgen, F.J., Weber, S.L., 2012 Monsoonal response to mid-Holocene orbital forcing in a high resolution GCM. Clim. Past 8, 723-740.

Braconnot, P., Harrison, S.P., Jouassaume, S., Hewitt, C.D., Kitoh, A., Kutzbach, J.E., Liu, Z. Otto-Bliesner, B., Syktus, J., Weber, S.L., 2004. Evaluation of PMIP coupled oceanatmosphere simulations of the mid-Holocene. In: Battarbee, R.W., Gasse, F., Stickley, C.E. (Eds.), Past Climate Variability Through Europe and Africa. Springer, Dordrecht, The Netherlands, pp. 515-533.

Braconnot, P., Otto-Bliesner, B., Harrison, S., Joussaume, S., Peterchmitt, J.Y., Abe-Ouchi, A Crucifix, M., Driesschaert, E., Fichefet, T., Hewitt, C.D., Kageyama, M., Kitoh, A., Laine, A., Loutre, M.F., Marti, O., Merkel, U., Ramstein, G., Valdes, P., Weber, S.L., Yu, Y., Zhao, Y., 2007a. Results of PMIP2 coupled simulations of the Mid-Holocene and Last Glacial Maximum - Part 1: experiments and large-scale features. Clim. Past 3, 261-277. 
Braconnot, P., Otto-Bliesner, B., Harrison, S., Joussaume, S., Peterchmitt, J.Y., Abe-Ouchi, A Crucifix, M., Driesschaert, E., Fichefet, T., Hewitt, C.D., Kageyama, M., Kitoh, A., Loutre M.F., Marti, O., Merkel, U., Ramstein, G., Valdes, P., Weber, L., Yu, Y., Zhao, Y., 2007b. Results of PMIP2 coupled simulations of the Mid-Holocene and Last Glacial Maximum - Part 2: feedbacks with emphasis on the location of the ITCZ and mid- and high latitudes heat budget. Clim. Past 3, 279-296.

Braconnot, P., Marzin, C., Gregoire, L., Mosquet, E., Marti, O., 2008. Monsoon response to changes in Earth's orbital parameters: comparisons between simulations of the Eemian and of the Holocene. Clim. Past 4, 281-294.

Breeze, P.S., Drake, N.A., Groucutt, H.S., Parton, A., Jennings, R.P., White, T.S., Clark-Balzan, L., Shipton, C., Scerri, E.M.L., Stimpson, C.M., Crassard, R., Hilbert, Y., Alsharekh, A., AlOmari, A., Petraglia, M.D., 2015. Remote sensing and GIS techniques for reconstructing Arabian palaeohydrology and identifying archaeological sites. Quat. Int. http://dx.doi. org/10.1016/j.quaint.2015.01.022 (in press).

Burns, S.J., Matter, A., Frank, N., Mangini, A., 1998. Speleothem-based paleoclimate record from northern Oman. Geology 26, 499-502.

Burns, S.J., Fleitmann, D., Matter, A., Neff, U., Mangini, A., 2001. Speleothem evidence from Oman for continental pluvial events during interglacial periods. Geology 29, 623-626.

Burrough, S.L., Thomas, D.S.G., 2009. Geomorphological contributions to palaeolimnology on the African continent. Geomorphology 103, 285-298.

Catlett, A.G., 2014. Pluvial Deposits in Mudawwara, Jordan and Their Implications for Mediterranean and Monsoonal Precipitation in the Levant. (M.Sc. Thesis), Miami Univ., Oxford, Ohio (32 pp.).

Catlett, G.A., Rech, J.A., Pigati, J.S., Al Kuisi, M., 2013. Paleoclimatic implications of pluvia deposits in Mudawwara, Jordan. Geol. Soc. Am. Abstr. Programs 45 (7), 680.

Charney, J.G., 1975. Dynamics of deserts and drought in Sahel. Q. J. R. Meteorol. Soc. 101, 193-202.

Chou, C., Neelin, J.D., 2003. Mechanisms limiting the northward extent of the northern summer monsoons over North America, Asia, and Africa. J. Clim. 16, 406-425.

Cleuziou, S., Inizan, M.-L., Marcolongo, B., 1992. Le peupelment pré-et protohistorique du systéme fluviatile fossile du Jawf-Hadaramawt au Yémen. Paléorient 18, 2-29.

Conroy, J.L., Overpeck, J.T., 2012. Regionalization of present-day precipitation in the greater monsoon region of Asia. J. Clim. 24, 4073-4095.

Crassard, R., Petraglia, M.D., Drake, N.A., Breeze, P., Gratuze, B., Alsharekh, A., Arbach, A. Groucutt, H.S., Khalidi, L., Michelsen, N., Robin, C.J., Schiettecatte, J., 2013. Middle palaeolithic and neolithic occupations around Mundafan Palaeolake, Saudi Arabia: implications for climate change and human dispersals. PLoS One 8 (7), e69665.

Davies, C.P., 2006. Holocene paleoclimates of southern Arabia from lacustrine deposits of the Dhamar highlands, Yemen. Quat. Res. 66, 454-464.

de Vries, A.J., Tyrlis, E., Edry, D., Krichak, S.O., Steil, B., Lelieveld, J., 2013. Extreme precipitation events in the Middle East: dynamics of the Active Red Sea Trough. J. Geophys. Res. Atmos. 118, 7087-7108.

Dima, I.M., Wallace, J.M., 2003. On the seasonality of the Hadley cell. J. Atmos. Sci. 60, $1522-1527$.

El-Moslimany, A.P., 1983. History of Climate and Vegetation in the Eastern Mediterranean and the Middle East From the Pleniglacial to the Mid-Holocene. University of Washington (PhD Thesis, 228 pp.).

El-Moslimany, A.P., 1990. Ecological significance of common nonarboreal pollen: examples from drylands of the Middle East. Rev. Palaeobot. Palynol. 64, 343-350.

ElNesr, M., Alazba, A., Abu-Zreig, M., 2010. Spatio-temporal variability of evapotranspiration over the Kingdom of Saudi Arabia. Appl. Eng. Agric. 26, 833-842.

Engel, M., Brückner, H., Pint, A., Wellbrock, K., Ginau, A., Voss, P., Grottker, M., Klasen, N., Frenzel, P., 2012. The early Holocene humid period in NW Saudi Arabia-sediments, microfossils, and palaeo-hydrological modeling. Quat. Int. 266, 131-141.

Enzel, Y., 1992. Flood frequency of the Mojave River and the formation of late Holocene playa lakes, southern California. The Holocene 2, 11-18.

Enzel, Y., Wells, S.G., 1997. Extracting Holocene paleohydrology and paleoclimatology information from modern extreme flood events: an example from southern California. Geomorphology 19, 203-226

Enzel, Y., Brown, W.J., Anderson, R.Y., McFadden, L.D., Wells, S.G., 1992. Short-duration Holocene lakes in the Mojave River drainage basin, southern California. Quat. Res. 38, 60-73.

Enzel, Y., Ely, L.L., Mishra, S., Ramesh, R., Amit, R., Lazar, B., Rajaguru, S.N., Baker, V.R., Sandler, A., 1999. High-resolution Holocene environmental changes in the Thar Desert, northwestern India. Science 284, 125-128.

Enzel, Y., Bookman, R., Sharon, D., Gvirtzman, H., Dayan, U., Ziv, B., Stein, M., 2003. Late Holocene climates of the Near East deduced from Dead Sea level variations and modem regional winter rainfall. Quat. Res. 60, 263-273.

Evan, A.T., Camargo, S.J., 2011. A climatology of Arabian Sea cyclonic storms. J. Clim. 24, $140-158$.

Findlater, J., 1969a. A major low-level air current near Indian Ocean during northern summer. Q. J. R. Meteorol. Soc. 95, 362-380.

Findlater, J., 1969b. Interhemispheric transport of air in lower troposphere over western Indian Ocean. Q. J. R. Meteorol. Soc. 95, 400-403.

Findlater, J., 1971. Mean monthly airflow at low levels over the Western Indian Ocean. Geophysical Memoirs 115. Meteorological Office, London, UK (53 pp.).

Findlater, J., 1977. Observational aspects of the low-level cross-equatorial jet stream of the western Indian Ocean. Pure Appl. Geophys. 115, 1251-1262.

Fleitmann, D., Burns, S.J., Mudelsee, M., Neff, U., Kramers, J., Mangini, A., Matter, A., 2003a. Holocene forcing of the Indian monsoon recorded in a stalagmite from Southern Oman. Science 300, 1737-1739.

Fleitmann, D., Burns, S.J., Neff, U., Mangini, A., Matter, A., 2003b. Changing moisture sources over the last 330,000 years in Northern Oman from fluid-inclusion evidence in speleothems. Quat. Res. 60, 223-232.

Fleitmann, D., Matter, A., Pint, J.J., Al-Shanti, M.A., 2004. The speleothemn record of climate change in Saudi Arabia. Jeddah, Saudi Arabia, Saudi Geological Survey OpenFile Report SGS-OF-2004-8 (40 pp.).
Fleitmann, D., Burns, S.J., Mangini, A., Mudelsee, M., Kramers, J., Villa, I., Neff, U., AlSubbary, A.A., Buettner, A., Hippler, D., Matter, A., 2007. Holocene ITCZ and Indian monsoon dynamics recorded in stalagmites from Oman and Yemen (Socotra). Quat. Sci. Rev. 26, 170-188.

Fleitmann, D., Burns, S.J., Pekala, M., Mangini, A., Al-Subbary, A., Al-Aowah, M., Kramers, J., Matter, A., 2011. Holocene and Pleistocene pluvial periods in Yemen, southern Arabia. Quat. Sci. Rev. 30, 783-787.

Frumkin, A., Bar-Matthews, M., Vaks, A., 2008. Paleoenvironment of Jawa basalt plateau, Jordan, inferred from calcite speleothems from a lava tube. Quat. Res. 70, 358-367.

Fuchs, M., Buerkert, A., 2008. A 20 ka sediment record from the Hajar Mountain range in $\mathrm{N}-\mathrm{Oman}$, and its implication for detecting arid-humid periods on the southeastern Arabian Peninsula. Earth Planet. Sci. Lett. 265, 546-558.

Gasse, F., 2000. Hydrological changes in the African tropics since the Last Glacial Maximum. Quat. Sci. Rev. 19, 189-211.

Gasse, F., Roberts, C.N., 2005. Late quaternary hydrologic changes in the arid and semiarid belt of northern Africa: implications for past atmospheric circulation. In: Diaz, H.F., Bradley, R.S. (Eds.), The Hadley Circulation: Present, Past and Future. Kluwer Academic Publishers, pp. 313-345.

Gasse, F., Street, F.A., 1978. Late Quaternary lake-level fluctuations and environments of the northern Rift Valley and Afar region (Ethiopia and Djibouti. Palaeogeogr. Palaeoclimatol. Palaeoecol. 24, 279-325.

Gennari, G., Rosenberg, T., Spezzaferri, S., Berger, J.-P., Fleitmann, D., Preusser, F., Al-Shanti, M., Matter, A., 2011. Faunal evidence of a Holocene pluvial phase in southern Arabia with remarks on the morphological variability of Helenina anderseni. J. Foraminifer. Res. 41, 248-259.

Ginau, A., Engel, M., Bruckner, H., 2012. Holocene chemical precipitates in the continental sabkha of Tayma (NW Saudi Arabia). J. Arid Environ. 84, 26-37.

Goudie, A.S., Colls, A., Stokes, S., Parker, A.I., White, K., Al-Farraj, A., 2000. Latest Pleistocene and Holocene dune construction at the north-eastern edge of the Rub' al-Khali, United Arab Emirates. Sedimentology 47, 1011-1021.

Halpern, D., Woiceshyn, P.M., 2001. Somali Jet in the Arabian Sea, El Nino, and India rainfall. J. Clim. 14, 434-441.

Herold, M., Lohmann, G., 2009. Eemian tropical and subtropical African moisture transport: an isotope modelling study. Clim. Dyn. 33, 1075-1088.

Hoelzmann, P., Gasse, F., Dupont, L.M., Salzmann, H., Staubwasser, M., Leuschner, D.C., Sirocko, F., 2004. Palaeoenvironmental changes in the arid and sub arid belt (SaharaSahel-Arabian Peninsula) from $150 \mathrm{kyr}$ to present. In: Battarbee, R.W., Gasse, F., Stickley, C.E. (Eds.), Past Climate Variability Through Europe and Africa. Springer, Dordrecht, The Netherlands, pp. 219-256.

Hudson, A., Quade, J., 2013. Long-term east-west asymmetry in monsoon intensification on the Tibetan Plateau. Geology 41, 351-354.

Hunter, R.E., Reiss, T.E., Chin, J.L., Anima, R.J., 1990. Coastal depositional and erosional effects of the 1985-1987 high lake levels in Lake Michigan. Open-File Report 90-272. U.S. Geological Survey, Washington, D.C.

Junginger, A., Roller, S., Olaka, L.A., Trauth, M.H., 2014. The effects of solar irradiation changes on the migration of the Congo Air Boundary and water levels of paleoake Suguta, Northern Kenya Rift, during the African Humid Period (15-5 ka BP). Palaeogeogr. Palaeoclimatol. Palaeoecol. 396, 1-16.

Kahana, R., Ziv, B., Enzel, Y., Dayan, U., 2002. Synoptic climatology of major floods in the Negev Desert, Israel. Int. J. Climatol. 22, 867-882.

Kohfeld, K.E., Harrison, S.P., 2000. How well can we simulate past climates? Evaluating the models using global palaeoenvironmental datasets. Quat. Sci. Rev. 19, 321-346.

Krishnamurti, T.N., Stefanova, L., Misra, V., 2013. Tropical Meteorology: An Introduction. Springer (423 pp.)

Kutzbach, J.E., Chen, G., Cheng, H., Edwards, R.L., Liu, Z., 2014. Potential role of winter rainfall in explaining increased moisture in the Mediterranean and Middle East during periods of maximum orbitally-forced insolation seasonality. Clim. Dyn. 42, 1079-1095.

Kwarteng, A.Y., Dorvlo, A., Vijaya Kumar, G.T., 2009. Analysis of a 27-year rainfall data (1977-2003) in the Sultanate of Oman. Int. J. Climatol. 29, 605-617.

Lézine, A.-M., 2009. Timing of vegetation changes at the end of the Holocene Humid Period in desert areas at the northern edge of the Atlantic and Indian monsoon systems. Compt. Rendus Geosci. 341, 750-759.

Lézine, A.M., Saliege, J.F., Robert, C., Wertz, F., Inizan, M.L., 1998. Holocene lakes from Ramlat as-Sab'atayn (Yemen) illustrate the impact of monsoon activity in Southern Arabia. Quat. Res. 50, 290-299.

Lézine, A.-M., Tiercelin, J.-J., Robert, C., Saliege, J.-F., Cleuziou, S., Inizan, M.-L., Braemer, F., 2007. Centennial to millennial-scale variability of the Indian monsoon during the early Holocene from a sediment, pollen and isotope record from the desert of Yemen. Palaeogeogr. Palaeoclimatol. Palaeoecol. 243, 235-249.

Lézine, A.-M., Robert, C., Cleuziou, S., Inizan, M.-L., Braemer, F., Saliege, J.-F., Sylvestre, F., Tiercelin, J.-J., Crassard, R., Mery, S., Charpentier, V., Steimer-Herbet, T., 2010. Climate change and human occupation in the Southern Arabian lowlands during the last deglaciation and the Holocene. Glob. Planet. Chang. 72, 412-428.

Litt, T., Ohlwein, C., Neumann, F.H., Hense, A., Stein, M., 2012. Holocene climate variability in the Levant from the Dead Sea pollen record. Quat. Sci. Rev. 49, 95-105.

Marzin, C., Braconnot, P., 2009. Variations of Indian and African monsoons induced by insolation changes at 6 and $9.5 \mathrm{kyr}$ BP. Clim. Dyn. 33, 215-231.

Matter, A., Neubert, E., Preusser, F., Rosenberg, T., Al-Wagdani, K., 2015. Palaeoenvironmental implications derived from lake and sabkha deposits of the southern Rub' al-Khali, Saudi Arabia and Oman. Quat. Int. http://dx.doi.org/10.1016/j.quaint. 2014.12.029 (in press)

McClure, H.A., 1976. Radiocarbon chronology of late Quaternary lakes in Arabian Desert. Nature 263, 755-756

McClure, H.A., 1984. Late Quaternary Palaeoenvironments of the Rub' al-Khali. (Ph.D. thesis), Department of Geography, University College London (245 pp.). 
Mifflin, M.D., Wheat, M.M., 1979. Pluvial lakes and estimated pluvial climates of Nevada Nevada Bureau of Mines and Geology Bull 94. University of Nevada, Reno (57 pp.).

Mischke, S., Ginat, H., Faerstein, G., Porat, N., Braun, P., Rech, Jason, 2016. Fossil-based reconstructions of ancient water bodies in the Levantine deserts. In: Enzel, Y., Bar-Yosef, O. (Eds.), Quaternary Environments, Climate Change, and Humans in the Levant. Cambridge University Press (accepted for publication).

Mitchell, T.D., Jones, P.D., 2005. An improved method of constructing a database of monthly climate observations and associated high-resolution grids. Int. J. Climatol. $25,693-712$.

Neff, U., Burns, S.J., Mangini, A., Mudelsee, M., Fleitmann, D., Matter, A., 2001. Strong coherence between solar variability and the monsoon in Oman between 9 and $6 \mathrm{kyr}$ ago. Nature 411, 290-293.

Overpeck, J., Anderson, D., Trumbore, S., Prell, W., 1996. The southwest Indian Monsoon over the last 18000 years. Clim. Dyn. 12, 213-225.

Parker, A.G., Goudie, A.S., 2008. Geomorphological and palaeoenvironmental investigations in the southeastern Arabian Gulf region and the implication for the archaeology of the region. Geomorphology 101, 458-470.

Parker, A.G., Eckersley, L., Smith, M.M., Goudie, A.S., Stokes, S., Ward, S., White, K., Hodson, M.J., 2004. Holocene vegetation dynamics in the northeastern Rub' al-Khali desert, Arabian Peninsula: a phytolith, pollen and carbon isotope study. J. Quat. Sci. 19, 665-676.

Peterson, L.C., Overpeck, J.T., Kipp, N.G., Imbrie, J., 1991. A high resolution late Quaternary upwelling record from the anoxic Cariaco Basin, Venezuela. Paleoceanography 6, 99-119.

Petraglia, M.D., Alsharekh, A.M., Crassard, R., Drake, N.A., Groucutt, H., Parker, A.G., Roberts, R.G., 2011. Middle Paleolithic occupation on a Marine Isotope Stage 5 lakeshore in the Nefud Desert, Saudi Arabia. Quat. Sci. Rev. 30, 1555-1559.

Petit-Maire, N., Carbonel, P., Reyss, J.L., Sanlaville, P., Abed, A., Bourrouilh, R., Fontugne, M., Yasin, S., 2010. A vast Eemian palaeolake in Southern Jordan $\left(29^{\circ} \mathrm{N}\right)$. Glob. Planet. Chang. 72, 368-373.

Pigati, J.S., Bright, J.E., Shanahan, T.M., Mahan, S.A., 2009. Late Pleistocene paleohydrology near the boundary of the Sonoran and Chihuahuan Deserts, southeastern Arizona, USA. Quat. Sci. Rev. 28, 286-300.

Pigati, J.S., Miller, D.M., Bright, J.E., Mahan, S.A., Nekola, J.C., Paces, J.B., 2011. Chronology, sedimentology, and microfauna of groundwater discharge deposits in the central Mojave Desert, Valley Wells, California. Geol. Soc. Am. Bull. 123, 2224-2239.

Pigati, J.S., Rech, J.A., Quade, J., Bright, J., 2014. Desert wetlands in the geologic record. Earth Sci. Rev. 132, 67-81.

Prasad, S., Enzel, Y., 2006. Holocene paleoclimates of India. Quat. Res. 66, 442-453.

Prell, W.L., 1984. Variations of monsoonal upwelling: a response to changing solar radiation. In: Hansen, J.E., Takahashi, T. (Eds.), Climate Processes and Climate Sensitivity. American Geophysical Union Geophysical Monograph 29. AGU, Washington, D.C. pp. 48-57.

Prell, W.L., Van Campo, E., 1986. Coherent response of Arabian Sea upwelling and pollen transport to late Quaternary monsoonal winds. Nature 323, 526-528.

Prell, W.L., Marvil, R.E., Luther, M.E., 1990. Variability in upwelling fields in the northwestern Indian Ocean 2. Data-model comparison at 9000 years B.P. Paleoceanography 5 , 447-457.

Prentice, I.C., Jolly, D., et al., 2000. Mid-Holocene and glacial-maximum vegetation geography of the northern continents and Africa. J. Biogeogr. 27, 507-519.

Preston, G.W., Thomas, D.S.G., Goudie, A.S., Atkinson, O.A.C., Leng, M.J., Hodson, M.J., Walkington, H., Charpentier, V., Méry, S., Borgi, F., Parker, A.G., 2015. A multi-proxy analysis of the Holocene humid phase from the United Arab Emirates and its implications for southeast Arabia's Neolithic populations. Quat. Int. http://dx.doi.org/10. 1016/j.quaint.2015.01.054 (in press).

Quade, J., 1986. Late Quaternary environmental changes in the upper Las Vegas Valley, Nevada. Quat. Res. 26, 340-357.

Quade, J., Pratt, W.L., 1989. Late Wisconsin groundwater discharge environments of the southwestern Indian Spring valley, southern Nevada. Quat. Res. 31, 351-370.

Quade, J., Mifflin, M.D., Pratt, W.L., McCoy, W., Burckle, L., 1995. Fossil spring deposits in the southern Great basin and their implications for changes in water-table levels near Yucca Mountain, Nevada, during Quaternary time. Geol. Soc. Am. Bull. 107, 213-230.

Quade, J., Forester, R.M., Pratt, W.L., Carter, C., 1998. Black mats, spring-fed streams, and late-glacial-age recharge in the southern Great Basin. Quat. Res. 49, 129-148.

Quade, J., Forester, R.M., Whelan, J.E., 2003. Late Quaternary paleohydrology and paleotemperature changes in southern Nevada. In: Enzel, Y., Wells, S.G., Lancaster, N. (Eds.), Paleoenvironments and Paleohydrology of the Mojave and southern Great Basin Deserts. Boulder, Colorado, Geol. Soc. Am. Special Paper 368, pp. $165-188$.

Quade, J., Rech, J.A., Betancourt, J.L., Latorre, C., Quade, B., Rylander, K.A., Fisher, T., 2008. Paleowetlands and regional climate change in the central Atacama Desert, northern Chile. Quat. Res. 69, 343-360.

Radies, D., Preusser, F., Matter, A., Mange, M., 2004. Eustatic and climatic controls on the development of the Wahiba Sand Sea, Sultanate of Oman. Sedimentology 51, 1359-1385.

Radies, D., Hasiotis, S.T., Preusser, F., Neubert, E., Matter, A., 2005. Paleoclimatic significance of Early Holocene faunal assemblages in wet interdune deposits of the Wahiba Sand Sea, Sultanate of Oman. J. Arid Environ. 62, 109-125.

Rech, J.A., Quade, J., Betancourt, J.L., 2002. Late Quaternary paleohydrology of the central Atacama Desert (lat 22 degrees-24 degrees S), Chile. Geol. Soc. Am. Bull. 114, 334-348.

Rech, J.A., Pigati, J.S., Quade, J., Betancourt, J.L., 2003. Re-evaluation of mid-Holocene deposits at Quebrada Puripica, northern Chile. Palaeogeogr. Palaeoclimatol. Palaeoecol. 194, 207-222.

Rech, J.A., Pigati, J.S., Al Kuisi, M., Bright, J., 2014. The rise and fall of late Pleistocene paleolakes in southern Jordan. Geol. Soc. Am. Abstr. Programs 45 (7), 680.
Rech, J.A., Ginat, A., Catlett, G.A., Mischke, S., Tully, E.W., Pigati, J.S., 2016. Pleistocene water bodies and associated geologic deposits in the southern Levant. In: Enzel, Y., BarYosef, O. (Eds.), Quaternary Environments, Climate Change, and Humans in the Levant. Cambridge University Press (accepted for publication).

Roberts, N., Wright Jr., H.E., 1993. Vegetational, lake-level, and climatic history of the Near East and southwest Asia. In: Wright Jr., H.E., Kutzbach, J.E., Webb III, T. Ruddiman, W.F., Street-Perrott, A.F., Bartlein, P.J. (Eds.), Global Climates Since the Last Glacial Maximum. University of Minnesota Press, Minneapolis, Minnesota, pp. 194-220.

Rodwell, M.J., Hoskins, B.J., 1996. Monsoons and the dynamics of deserts. Q. J. R. Meteorol. Soc. $122,1385-1404$.

Rosen, M.R., 1991. Sedimentologic and geochemical constrains on the hydrologic evolution of Bristol Dry Lake, California, USA. Palaeogeogr. Palaeoclimatol. Palaeoecol. 8 229-257.

Rosen, M.R., 1994. The importance of groundwater in playas: a review of playa classifications and the sedimentology and hydrology of playas. Geol. Soc. Am. Spec. Pap. 289, $1-18$.

Rosenberg, T.M., Preusser, F., Fleitmann, D., Schwalb, A., Penkman, K., Schmid, T.W., AlShanti, M.A., Kadi, K., Matter, A., 2011. Humid periods in southern Arabia: windows of opportunity for modern human dispersal. Geology 39, 1115-1118.

Rosenberg, T.M., Preusser, F., Risberg, J., Plikk, A., Kadi, K.A., Matter, A., Fleitmann, D., 2013. Middle and Late Pleistocene humid periods recorded in palaeolake deposits of the Nafud desert, Saudi Arabia. Quat. Sci. Rev. 70, 109-123.

Rubin, S., Ziv, B., Paldor, N., 2007. Tropical plumes over eastern North Africa as a source of rain in the Middle East. Mon. Weather Rev. 135, 4135-4148.

Scholte, P., De Geest, P., 2010. The climate of Socotra Island (Yemen): a first-time assessment of the timing of the monsoon wind reversal and its influence on precipitation and vegetation patterns. J. Arid Environ. 74, 1507-1515.

Schulz, E., Whitney, J.W., 1986a. Upper Pleistocene and Holocene lakes in the An Nafud, Saudi Arabia. Hydrobiologia 143, 175-190.

Schulz, E., Whitney, J.W., 1986b. Vegetation in north-central Saudi Arabia. J. Arid Environ. $10,175-186$.

Shakun, J.D., Burns, S.J., Fleitmann, D., Kramers, J., Matter, A., Al-Subary, A., 2007. A highresolution, absolute-dated deglacial speleothem record of Indian Ocean climate from Socotra Island, Yemen. Earth Planet. Sci. Lett. 259, 442-456.

Sirocko, F., Sarnthein, M., Erlenkeuser, H., Lange, H., Arnold, M., Duplessy, J.C., 1993. Century-scale events in monsoonal climate over the past 24,000 years. Nature 364 , 322-324.

Slingo, J., Spencer, H., Hoskins, B., Berrisford, P., Black, E., 2005. The meteorology of the Western Indian Ocean, and the influence of the East African Highlands. Phil. Trans. R. Soc. A 363, 25-42.

Sorman, A.U., Abdulrazzak, M.J., 1995. Estimation of actual evaporation using precipitation and soil moisture records in arid climates. Hydrol. Process. 9, 729-741.

Staubwasser, M., 2006. An overview of Holocene South Asian monsoon records monsoon domains and regional contrasts. J. Geol. Soc. India 68, 433-446.

Staubwasser, M., Weiss, H., 2006. Holocene climate and cultural evolution in late prehistoric-early historic West Asia - introduction. Quat. Res. 66, 372-387.

Stine, S., Stine, M., 1990. A record from Lake Cardiel of climate change in southern South America. Nature 345, 704-708.

Street-Perrott, F.A., Harrison, S.P., 1985. Lake levels and climate reconstruction. In: Hecht, A.D. (Ed.), Paleoclimate Analysis and Modeling. Wiley, New York, pp. 291-340.

Street-Perrott, F.A., Perrott, R.A., 1993. Holocene vegetation, lake levels, and climate of Africa. In: Wright Jr., H.E., Kutzbach, J.E., Webb III, T., Ruddiman, W.F., StreetPerrott, A.F., Bartlein, P.J. (Eds.), Global Climates Since the Last Glacial Maximum. University of Minnesota Press, Minneapolis, Minnesota, pp. 318-356.

Street-Perrott, F.A., Marchand, D.S., Roberts, N., Harisson, S.P., 1989. Global lake-leve variations from 18,000 to 0 years ago: a paleoclimatic analysis. U.S. Department of Energy Technical Report 46, Washington, D.C. 20545 (Distributed by National Technical Information Service, Springfield, VA 22161).

Su, H., Neelin, J.D., 2005. Dynamical mechanisms for African monsoon changes during the mid-Holocene. J. Geophys. Res. Atmos. 110 (paper D19105)

Subyani, A.M., Al-Dakheel, A.M., 2009. Multivariate geostatistical methods of mean annual and seasonal rainfall in southwest Saudi Arabia. Arab. J. Geosci. 2, 19-27.

Sultan, M., Sturchio, N., Al Sefry, S., Milewski, A., Becker, R., Nasr, I., Sagintayev, Z., 2008 Geochemical, isotopic, and remote sensing constraints on the origin and evolution of the Rub Al Khali aquifer system, Arabian Peninsula. J. Hydrol. 356, 70-83.

Tanaka, H.L., Ishizaki, N., Kitoh, A., 2004. Trend and interannual variability of Walker monsoon and Hadley circulations defined by velocity potential in the upper troposphere. Tellus Ser. A Dyn. Meteorol. Oceanogr. 56, 250-269.

Texier, D., de Noblet, N., Braconnot, P., 2000. Sensitivity of the African and Asian monsoons to mid-Holocene insolation and data-inferred surface changes. J. Clim. 13, 164-181.

Thompson, T.A., Baedke, S.J., 1995. Beach-ridge development in Lake Michigan: shoreline behavior in response to quasi-periodic lake-level events. Mar. Geol. 129, 163-174.

Tubi, A., Dayan, U., 2014. Tropical plumes over the Middle East: climatology and synoptic conditions. Atmos. Res. 145-146, 168-181.

Turner, A., Annamalai, H., 2012. Climate change and the South Asia summer monsoon. Nat. Clim. Chang. 2, 587-595.

Vaks, A., Bar-Matthews, M., Matthews, A., Ayalon, A., Frumkin, A., 2010. Middle-Late Quaternary paleoclimate of northern margins of the Saharan-Arabian Desert: reconstruction from speleothems of Negev Desert, Israel. Quat. Sci. Rev. 29, 2647-2662.

Van Rampelbergh, M., Fleitmann, D., Verheyden, S., Cheng, H., Edwards, L., De Geest, P., De Vleeschouwer, D., Burns, S.J., Matter, A., Claeys, P., Keppens, E., 2013. Mid- to late Holocene Indian Ocean Monsoon variability recorded in four speleothems from Socotra Island, Yemen. Quat. Sci. Rev. 65, 129-142.

Waliser, D.E., Gautier, C., 1993. A satellite-derived climatology of the ITCZ. J. Clim. 6, 2162-2174. 
Waliser, D.E., Somerville, R.C.J., 1994. Preferred latitudes of the intertropical convergence zone. J. Atmos. Sci. 51, 1619-1639.

Wanner, H., Beer, J., Buetikofer, J., Crowley, T.J., Cubasch, U., Flueckiger, J., Goosse, H. Grosjean, M., Joos, F., Kaplan, J.O., Kuettel, M., Mueller, S.A., Prentice, I.C., Solomina, O., Stocker, T.F., Tarasov, P., Wagner, M., Widmann, M., 2008. Mid- to Late Holocene climate change: an overview. Quat. Sci. Rev. 27, 1791-1828.

Wellbrock, K., Grottker, M., 2010. Reconstruction of mid-Holocene climate conditions for north-western Arabian oasis Tayma. 4th International Conference on Water Resources and Arid Environments (ICWRAE 4), Riyadh, Saudi Arabia, pp. 694-703 (http://www.icwrae-psipw.org/images/stories/2010/New_Technologies/5/index. html).

Wellbrock, K., Voss, P., Grottker, M., 2011. Reconstruction of mid-Holocene climate conditions for north-western Arabian oasis Tayma. Int. J. Water Resour. Arid Environ. 1, $200-209$.

Weyhenmeyer, C.E., Burns, S.J., Waber, H.N., Werner, A.-H., Kipfer, R., Loosli, H.H., Matter, A., 2000. Cool glacial temperatures and changes in moisture source recorded in Oman groundwaters. Science 287, 842-845.

Weyhenmeyer, C.E., Burns, S.J., Waber, H.N., Macumber, P.G., Matter, A., 2002. Isotope study of moisture sources, recharge areas, and groundwater flow paths within the eastern Batinah coastal plain, Sultanate of Oman. Water Resour. Res. 38 (1184), 2-1-2-22.

Whitney, J.W., Faulkender, D.J., Rubin, M., 1983. The environmental history and present condition of Saudi Arabia's northern sand seas. U.S. Geological Survey Open-File Report 83-749 (39 pp.).

Wilkinson, T.J., 2003. The organization of settlement in highland Yemen during the Bronze and Iron Age. Proc. Semin. Arab. Stud. 33, 157-168.

Yechieli, Y., Wood, W., 2002. Hydrogeologic processes in saline systems: playas, sebkhas, and saline lakes. Earth-Sci. Rev. 58, 343-365.

Zhao, Y., Braconnot, P., Marti, O., Harrison, S.P., Hewitt, C., Kitoh, A., Liu, Z., Mikolajewicz, U., Otto-Bliesner, B., Weber, S.L., 2005. A multi-model analysis of the role of the ocean on the African and Indian monsoon during the mid-Holocene. Clim. Dyn. 25, 777-800.

Ziv, B., 2001. A subtropical rainstorm associated with a tropical plume over Africa and the Middle-East. Theor. Appl. Climatol. 69, 91-102.

Ziv, B., Saaroni, H., Alpert, P., 2004. The factors governing the summer regime of the eastern Mediterranean. Int. J. Climatol. 24, 1859-1871. 